Article

\title{
Generalized Contractive Mappings and Related Results in $b$-Metric Like Spaces with an Application
}

\author{
Hasanen A. Hammad ${ }^{1}$ (D) and Manuel De la Sen ${ }^{2, *(D)}$ \\ 1 Department of Mathematics, Faculty of Science, Sohag University, Sohag 82524, Egypt; \\ h_elmagd89@yahoo.com \\ 2 Institute of Research and Development of Processes, University of the Basque Country, \\ 48940 Leioa (Bizkaia), Spain \\ * Correspondence: manuel.delasen@ehu.eus; Tel.:+3-494-601-2548
}

Received: 12 April 2019; Accepted: 11 May 2019; Published: 14 May 2019

\begin{abstract}
In this article, a general contractive mapping is presented and some fixed point results in complete $b$-metric-like spaces are studied. The results obtained here extend and improve some related results in the literature. Also, new common fixed point results for a graphic contraction mappings are proved. Some comparative examples are given to support the obtained results. Moreover, an analytical solution of an integral equation has been presented as an application.
\end{abstract}

Keywords: a $b$-metric-like space; general contractive mappings; graphic contraction mappings; an integral equation

MSC: 47H04; 47H05

\section{Introduction}

Fixed point theory plays an important role in applications of many branches of mathematics. Within the past thirty years several generalizations of a metric space have been made.

Problems in nonlinear analysis are solved by a popular tool called the Banach contraction principle. A lot of publications are devoted to the study and solutions of many practical and theoretical problems by using this principle [1-6]. One of the interesting generalizations of this basic principle was given by Bakhtin [7] (and also Czerwik [8]) by introducing the concept of $b$-metric spaces. Following the initial paper of Czerwik [8], a number of researchers in nonlinear analysis investigated the topology of the paper and proved several fixed point theorems in the context of complete $b$-metric spaces, (for example, see [9-15]).

The notion of a $b$-metric-like presented by Alghamdi et al. [16] as a generalization of a $b$-metric. They discussed some related fixed point consequences concerning with this space. Recently, Hussain et al. [17] examined topological structure of this space and presented some fixed point results in $b$-metric-like space. A lot of results on fixed points of mappings via certain contractive conditions in mention spaces have been done (for example, see [18-24]).

Many authors generalized fixed point theory in various directions either by using generalized contractions or by using more general spaces. Via these directions, in this article, we introduce generalized contractive mappings (so-called $\beta_{q, \phi}^{s, \psi}$-contraction mappings) and prove some new results on common fixed points. The obtained results generalize some classical common fixed point theorems in the literature. Finally, some common fixed point results for $(s, q)$-graphic contraction mappings, illustrative examples and an application to nonlinear integral equation are presented to justify the obtained results. 


\section{Preliminaries}

This part is devoted to present some definitions and basic notions of metric-like and $b$-metric-like spaces.

Definition 1 ([17]). Let $\Omega$ be a nonempty set. A mapping $\omega: \Omega \times \Omega \rightarrow[0,+\infty)$ is said to be a metric-like if the following three conditions hold for all $\kappa, \tau, \mu \in \Omega$ :

$\left(\omega_{1}\right) \omega(\kappa, \tau)=0 \Rightarrow \kappa=\tau$;

$\left(\omega_{2}\right) \omega(\kappa, \tau)=\omega(\tau, \kappa)$

$\left(\omega_{3}\right) \omega(\kappa, \mu) \leqslant \omega(\kappa, \tau)+\omega(\tau, \mu)$.

In this case, the pair $(\Omega, \omega)$ is called a metric-like space.

Definition 2 ([25]). A b-metric-like on a nonempty set $\Omega$ is a function $\omega: \Omega \times \Omega \rightarrow[0,+\infty)$ such that for all $\kappa, \tau, \mu \in \Omega$, the following three conditions hold:

$\left(\omega_{1}\right) \omega_{b}(\kappa, \tau)=0 \Rightarrow \kappa=\tau$;

$\left(\omega_{2}\right) \omega_{b}(\kappa, \tau)=\omega_{b}(\tau, \kappa)$

$\left(\omega_{3}\right) \omega(\kappa, \mu) \leqslant s\left[\omega_{b}(\kappa, \tau)+\omega_{b}(\tau, \mu)\right]$

In this case, the pair $\left(\Omega, \omega_{b}\right)$ is called a b-metric-like space (with a constant $s \geqslant 1$ ).

In a $b$-metric-like space $\left(\Omega, \omega_{b}\right)$, if $\kappa, \tau \in \Omega$ and $\omega_{b}(\kappa, \tau)=0$, then $\kappa=\tau$, and the converse is not true in general.

Example 1. Let $\Omega=\{0,1,2\}$ and let

$$
\omega_{b}(\kappa, \tau)= \begin{cases}3, & \kappa=\tau=0 \\ \frac{1}{3}, & \text { otherwise. }\end{cases}
$$

Then $\left(\Omega, \omega_{b}\right)$ is a b-metric-like space with the constant $s=3$.

Example 2. If $\Omega=\mathbb{R}$, then $\omega_{b}(\kappa, \tau)=|\kappa|+|\tau|$ defines a metric-like on $\Omega$.

Example 3. Let $\Omega=[0,+\infty), q>1$ be a constant, and $\omega_{b}: \Omega \times \Omega \rightarrow[0,+\infty)$ be defined by

$$
\omega_{b}(\kappa, \tau)=(\kappa+\tau)^{q}, \quad \forall \kappa, \tau \in \Omega
$$

Then $\left(\Omega, \omega_{b}\right)$ is a $b$-metric-like space with coefficient $s=2^{q-1}$.

Example 4. Let $\left(\Omega, \omega_{b}\right)$ be a metric-like space and $\omega_{b}(\kappa, \tau)=(\omega(\kappa, \tau))^{q}$, where $q>1$ is a real number, then $\left(\Omega, \omega_{b}\right)$ is a $b$-metric-like space with coefficient $s=2^{q-1}$, this follows immediately by the fact that $(a+b)^{q} \leqslant 2^{q-1}\left(a^{q}+b^{q}\right)$.

By Example 4, we can get:

Example 5. Let $\Omega=[0,1]$. Then the mapping $\omega_{b 1}: \Omega \times \Omega \rightarrow[0,+\infty)$ defined by $\omega_{b 1}(\kappa, \tau)=(\kappa+\tau-\kappa \tau)^{q}$ is $b$-metric-like on $\Omega$ with coefficient $s=2^{q-1}$, where $q>1$ is a real number.

Example 6. Let $\Omega=\mathbb{R}$. Then the mappings $\omega_{i}: \Omega \times \Omega \rightarrow[0,+\infty)(i \in\{2,3,4\})$, defined by

$$
\begin{aligned}
& \omega_{b 2}(\kappa, \tau)=(|\kappa|+|\tau|+a)^{q}, \\
& \omega_{b 3}(\kappa, \tau)=(|\kappa-b|+|\tau-b|)^{q} \\
& \omega_{b 4}(\kappa, \tau)=\left(\kappa^{2}+\tau^{2}\right)^{q} .
\end{aligned}
$$


are $b$-metric-like on $\Omega$, where $q>1, a \geqslant 0$ and $b \in \mathbb{R}$.

Definition 3 ([25]). Let $\left\{x_{n}\right\}$ be a sequence on a metric-like space $\left(\Omega, \omega_{b}\right)$ with coefficient s.

(i) If $\lim _{m, n \rightarrow \infty} \omega_{b}\left(\kappa_{n}, \kappa\right)=\omega_{b}(\kappa, \kappa)$, then the sequence $\left\{\kappa_{n}\right\}$ is said to be convergent to $\kappa$;

(ii) The sequence $\left\{\kappa_{n}\right\}$ is said to be a Cauchy sequence in $\left(\Omega, \omega_{b}\right)$ If $\lim _{m, n \rightarrow \infty} \omega\left(\kappa_{m}, \kappa_{n}\right)$ exists and is finite;

(iii) $\left(\kappa, \omega_{b}\right)$ is said to be a complete b-metric-like space if for every Cauchy sequence $\left\{\kappa_{n}\right\}$ in $\Omega$, there exists an $\kappa \in \Omega$, such that $\lim _{m, n \rightarrow \infty} \omega_{b}\left(\kappa_{m}, \kappa_{n}\right)=\omega_{b}(\kappa, \kappa)=\lim _{n \rightarrow \infty} \omega_{b}\left(\kappa_{n}, \kappa\right)$.

Remark 1. In a b-metric-like space the limit of a sequence need not be unique and a convergent sequence need not be a Cauchy sequence.

To show this remark, we give the following example

Example 7. Let $\Omega=[0,+\infty)$. Define a function $\omega_{b}: \Omega \times \Omega \rightarrow \Omega$ by $\omega_{b}(\kappa, \tau)=(\max \{\kappa, \tau\})^{2}$. Then $\left(\Omega, \omega_{b}\right)$ is a b-metric-like space with a coefficient $s=2$. Suppose that

$$
\left\{\kappa_{n}\right\}=\left\{\begin{array}{ll}
0 & \text { when } n \text { is odd } \\
1 & \text { when } n \text { is even }
\end{array} .\right.
$$

For $\kappa \geqslant 1, \lim _{n \rightarrow \infty} \omega_{b}\left(\kappa_{n}, \kappa\right)=\lim _{n \rightarrow \infty}\left(\max \left\{\kappa_{n}, \kappa\right\}\right)^{2}=\kappa^{2}=\omega_{b}(\kappa, \kappa)$. Therefore, it is a convergent sequence and $\kappa_{n} \rightarrow \kappa$ for all $\kappa \geqslant 1$. That is, limit of the sequence is not unique. Also, $\lim _{m, n \rightarrow \infty} \omega\left(\kappa_{m}, \kappa_{n}\right)$ does not exist. Thus, it is not a Cauchy sequence.

Lemma 1 ([26]). Let $T: \Omega \rightarrow \Omega$ be a nonlinear self-mapping on a b-metric-like space $\left(\Omega, \omega_{b}\right)$ with coefficient s. Consider $T$ is continuous at $\eta \in \Omega$. Then for all sequences $\left\{\kappa_{n}\right\}$ in $\Omega$ such that $\kappa_{n} \rightarrow \eta$, we get $T \kappa_{n} \rightarrow T \eta$ that is

$$
\lim _{n \rightarrow \infty} \omega_{b}\left(T \kappa_{n}, T \eta\right)=\omega_{b}(T \eta, T \eta)
$$

The proof of the following lemma is obvious.

Lemma 2. Suppose that $\left(\Omega, \omega_{b}\right)$ be a b-metric-like space with coefficient $s \geqslant 1$. Then

(i) If $\omega_{b}(\kappa, \tau)=0$, then $\omega_{b}(\kappa, \kappa)=\omega_{b}(\kappa, \tau)=0$;

(ii) If $\left\{\kappa_{n}\right\}$ is a sequence such that $\lim _{n \rightarrow \infty} \omega_{b}\left(\kappa_{n}, \kappa_{n+1}\right)=0$ then, we can write

$$
\lim _{n \rightarrow \infty} \omega_{b}\left(\kappa_{n}, \kappa_{n}\right)=\lim _{n \rightarrow \infty} \omega_{b}\left(\kappa_{n+1}, \kappa_{n+1}\right)=0 ;
$$

(iii) if $\kappa \neq \tau$, then $\omega_{b}(\kappa, \tau)>0$.

Lemma 3 ([27]). Let $\left\{\tau_{n}\right\}$ be a sequence on a complete b-metric-like space $\left(\Omega, \omega_{b}\right)$ with parameter $s \geqslant 1$ such that

$$
\lim _{n \rightarrow \infty} \omega_{b}\left(\tau_{n}, \tau_{n+1}\right)=0 .
$$

If $\lim _{n, m \rightarrow \infty} \omega_{b}\left(\tau_{n}, \tau_{m}\right) \neq 0$, there exists an $\varepsilon>0$ and sequences $\{m(\ell)\}_{\ell=1}^{\infty}$ and $\{n(\ell)\}_{\ell=1}^{\infty}$ of positive integers with $n_{\ell}>m_{\ell}>\ell$ such that

$$
\begin{aligned}
\omega_{b}\left(\tau_{n_{\ell}}, \tau_{m_{\ell}}\right) & \geqslant \varepsilon, \omega_{b}\left(\tau_{m_{\ell}}, \tau_{n_{\ell}-1}\right)<\varepsilon, \frac{\varepsilon}{s^{2}} \leqslant \lim \sup _{n \rightarrow \infty} \omega_{b}\left(\tau_{n_{\ell}-1}, \tau_{m_{\ell}-1}\right) \leqslant \varepsilon s, \\
\frac{\varepsilon}{s} & \leqslant \lim \sup _{n \rightarrow \infty} \omega_{b}\left(\tau_{n_{\ell}-1}, \tau_{m_{\ell}}\right) \leqslant \varepsilon, \frac{\varepsilon}{s} \leqslant \lim \sup _{n \rightarrow \infty} \omega_{b}\left(\tau_{n_{\ell}}, \tau_{m_{\ell}-1}\right) \leqslant \varepsilon s^{2} .
\end{aligned}
$$


Definition 4 ([28]). The nonlinear self-mappings $T, S: \Omega \rightarrow \Omega$ are weakly compatible if $T S \kappa=S T \kappa$, whenever $S \kappa=T \kappa$.

Proposition 1 ([29]). Let $T$ and $S$ be weakly compatible self-maps of a nonempty set $\Omega$. If $T$ and $S$ have a unique point of coincidence $\eta=S \xi=T \xi$, then $\eta$ is the unique common fixed point of $T$ and $S$.

\section{An $\beta_{q, \phi}^{s, \psi}$-Contraction Mapping}

This section is prepared to introduce $\beta_{q, \phi}^{s, \psi}$-contraction mappings and obtained some common fixed point results for such class of contractions in the framework of $b$-metric-like spaces.

Let $\Psi, \Phi$ denote the class of functions $\psi, \phi:[0, \infty) \rightarrow[0, \infty)$ (respectively) satisfying the following conditions:

- $\psi$ is non-decreasing, continuous function and $\psi(t)=0$, if $t=0$;

- $\phi$ is a lower semi-continuous and $\phi(t)=0$, if $t=0$.

Definition 5. Let $\left(\Omega, \omega_{b}\right)$ be a b-metric-like space with parameter $s \geqslant 1$. Let the constants $q \geqslant 2$ and $\beta \in[0,1)$. The nonlinear self-mappings $T, S: \Omega \rightarrow \Omega$ are called $\beta_{q, \phi}^{s, \psi}$-contraction mappings if for all $\kappa, \tau \in \Omega$

$$
\psi\left(2 s^{q} \omega_{b}(T \kappa, T \tau)\right) \leqslant \beta\left[\psi\left(M_{\omega_{b}}(\kappa, \tau)\right)-\phi\left(M_{\omega_{b}}(\kappa, \tau)\right)\right],
$$

where $\psi \in \Psi, \phi \in \Phi$ and

$$
M_{\omega_{b}}(\kappa, \tau)=\max \left\{\omega_{b}(S \kappa, S \tau), \omega_{b}(S \kappa, T \tau), \omega_{b}(S \tau, T \kappa), \frac{\omega_{b}(S \kappa, T \kappa)+\omega_{b}(T \tau, S \tau)}{4 s}\right\}
$$

Now we begin with our first result.

Theorem 1. Let $\left(\Omega, \omega_{b}\right)$ be a complete b-metric-like space with the constant $s \geqslant 1$, and $T, S: \Omega \rightarrow \Omega$ be mappings satisfy the following conditions:

(i) $T(\Omega) \subset S(\Omega)$;

(ii) the pair $(S, T)$ is an $\beta_{q, \phi}^{s, \psi}$-contraction;

then, $T$ and $S$ have a point of coincidence in $\Omega$.

(iii) Moreover, if $T$ and $S$ are weakly compatible, then $T$ and $S$ have a unique common fixed point in $\Omega$.

Proof. Let $\kappa_{\circ}$ be an arbitrary point in $\Omega$. Since $T(\Omega) \subset S(\Omega)$, there exists $\kappa_{1} \in \Omega$ such that $T \kappa_{\circ}=S \kappa_{1}$. By continuing this process inductively, we get a sequence $\left\{\kappa_{n}\right\}$ in $\Omega$ such that

$$
\tau_{n}=T \kappa_{n}=S \kappa_{n+1} .
$$

If $\omega_{b}\left(\tau_{n_{\circ}}, \tau_{n_{\circ}+1}\right)=0$ for some $n_{\circ} \in \mathbb{N}$, then we have $\tau_{n_{\circ}}=\tau_{n_{\circ}+1}$ i.e., $\tau_{n_{\circ}}$ is a common fixed point of the pair $(T, S)$ and the proof is finished.

Now, let for all $n_{\circ} \in \mathbb{N}, \omega_{b}\left(\tau_{n+1}, \tau_{n}\right)>0$.

By (2), one can get 


$$
\begin{aligned}
& \psi\left(2 s \omega_{b}\left(\tau_{n+1}, \tau_{n}\right)\right) \leqslant \psi\left(2 s^{q} \omega_{b}\left(\tau_{n+1}, \tau_{n}\right)\right) \\
& =\psi\left(2 s^{q} \omega_{b}\left(T \kappa_{n+1}, T \kappa_{n}\right)\right) \\
& \leqslant \beta \psi\left(M_{\omega_{b}}\left(\kappa_{n+1}, \kappa_{n}\right)\right)-\beta \phi\left(M_{\omega_{b}}\left(\kappa_{n+1}, \kappa_{n}\right)\right) \\
& =\beta \psi\left(\max \left\{\begin{array}{c}
\omega_{b}\left(S \kappa_{n+1}, S \kappa_{n}\right), \omega_{b}\left(S \kappa_{n+1}, T \kappa_{n}\right), \omega_{b}\left(S \kappa_{n}, T \kappa_{n+1}\right) \\
\frac{\omega_{b}\left(S \kappa_{n+1}, T \kappa_{n+1}\right)+\omega_{b}\left(T \kappa_{n}, S \kappa_{n}\right)}{4 s}
\end{array}\right\}\right) \\
& -\beta \phi\left(\max \left\{\begin{array}{c}
\omega_{b}\left(S \kappa_{n+1}, S \kappa_{n}\right), \omega_{b}\left(S \kappa_{n+1}, T \kappa_{n}\right), \omega_{b}\left(S \kappa_{n}, T \kappa_{n+1}\right) \\
\frac{\omega_{b}\left(S \kappa_{n+1}, T \kappa_{n+1}\right)+\omega_{b}\left(T \kappa_{n}, S \kappa_{n}\right)}{4 s}
\end{array}\right\}\right) \\
& =\beta \psi\left(\max \left\{\omega_{b}\left(\tau_{n}, \tau_{n-1}\right), \omega_{b}\left(\tau_{n}, \tau_{n}\right), \omega_{b}\left(\tau_{n-1}, \tau_{n+1}\right), \frac{\omega_{b}\left(\tau_{n}, \tau_{n+1}\right)+\omega_{b}\left(\tau_{n}, \tau_{n-1}\right)}{4 s}\right\}\right) \\
& -\beta \phi\left(\max \left\{\omega_{b}\left(\tau_{n}, \tau_{n-1}\right), \omega_{b}\left(\tau_{n}, \tau_{n}\right), \omega_{b}\left(\tau_{n-1}, \tau_{n+1}\right), \frac{\omega_{b}\left(\tau_{n}, \tau_{n+1}\right)+\omega_{b}\left(\tau_{n}, \tau_{n-1}\right)}{4 s}\right\}\right) \\
& \leqslant \beta \psi\left(\max \left\{\begin{array}{c}
\omega_{b}\left(\tau_{n}, \tau_{n-1}\right), 2 s \omega_{b}\left(\tau_{n}, \tau_{n-1}\right), s\left[\omega_{b}\left(\tau_{n-1}, \tau_{n}\right)+\omega_{b}\left(\tau_{n}, \tau_{n+1}\right)\right], \\
\frac{\omega_{b}\left(\tau_{n}, \tau_{n+1}\right)+\omega_{b}\left(\tau_{n-1}, \tau_{n}\right)}{4 s}
\end{array}\right\}\right) \\
& -\beta \phi\left(\max \left\{\begin{array}{c}
\omega_{b}\left(\tau_{n}, \tau_{n-1}\right), 2 s \omega_{b}\left(\tau_{n}, \tau_{n-1}\right), s\left[\omega_{b}\left(\tau_{n-1}, \tau_{n}\right)+\omega_{b}\left(\tau_{n}, \tau_{n+1}\right)\right], \\
\frac{\omega_{b}\left(\tau_{n}, \tau_{n+1}\right)+\omega_{b}\left(\tau_{n-1}, \tau_{n}\right)}{4 s}
\end{array}\right\}\right) .
\end{aligned}
$$

If $\omega_{b}\left(\tau_{n}, \tau_{n-1}\right) \leqslant \omega_{b}\left(\tau_{n}, \tau_{n+1}\right)$ for some $n \in \mathbb{N}$, then by (3), we can get

$$
\begin{aligned}
\psi\left(2 s \omega_{b}\left(\tau_{n+1}, \tau_{n}\right)\right) & \leqslant \beta \psi\left(2 s \omega_{b}\left(\tau_{n+1}, \tau_{n}\right)\right)-\beta \phi\left(2 s \omega_{b}\left(\tau_{n+1}, \tau_{n}\right)\right) \\
& <\psi\left(2 s \omega_{b}\left(\tau_{n+1}, \tau_{n}\right)\right)-\phi\left(2 s \omega_{b}\left(\tau_{n+1}, \tau_{n}\right)\right)
\end{aligned}
$$

According to definition of $\psi$ and $\phi$, the above inequality gives $\omega_{b}\left(\tau_{n+1}, \tau_{n}\right)=0$, which is a contradiction, since we have supposed $\omega_{b}\left(\tau_{n+1}, \tau_{n}\right)>0$. Hence, for all $n \in \mathbb{N}$

$$
\psi\left(2 s \omega_{b}\left(\tau_{n+1}, \tau_{n}\right)\right) \leqslant \psi\left(2 s^{q} \omega_{b}\left(\tau_{n+1}, \tau_{n}\right)\right) \leqslant \beta \psi\left(2 s \omega_{b}\left(\tau_{n}, \tau_{n-1}\right)\right)-\beta \phi\left(2 s \omega_{b}\left(\tau_{n}, \tau_{n-1}\right)\right) .
$$

Hence,

$$
\omega_{b}\left(\tau_{n+1}, \tau_{n}\right)<\omega_{b}\left(\tau_{n}, \tau_{n-1}\right)
$$

that is, a sequence $\left\{\omega_{b}\left(\tau_{n+1}, \tau_{n}\right)\right\}$ is decreasing and bounded below. Thus there exists $r \geqslant 0$ such that

$$
\lim _{n \rightarrow \infty} \omega_{b}\left(\tau_{n+1}, \tau_{n}\right)=r
$$

Now, we proof $r=0$ by a contradiction. Assume that $r>0$. Taking limit as $n \rightarrow \infty$ in (4), using (5), since $\beta \in[0,1)$ and by the properties of $\psi$ and $\phi$, we have

$$
\psi(2 s r) \leqslant \beta(\psi(2 s r)-\phi(2 s r))<\psi(2 s r)-\phi(2 s r)
$$

This is a contradiction. Hence

$$
\lim _{n \rightarrow \infty} \omega_{b}\left(\tau_{n+1}, \tau_{n}\right)=0
$$

In the next step, we claim that

$$
\lim _{n, m \rightarrow \infty} \omega_{b}\left(\tau_{n}, \tau_{m}\right)=0 .
$$

Let if possible, $\lim _{n, m \rightarrow \infty} \omega_{b}\left(\tau_{n}, \tau_{m}\right) \neq 0$, then by Lemma 3 , there exist $\varepsilon>0$ and sequences $\{m(\ell)\}$ and $\{n(\ell)\}$ of positive integers with $n_{\ell}>m_{\ell}>\ell$ such that $\omega_{b}\left(\kappa_{n_{\ell}}, \kappa_{m_{\ell}}\right) \geqslant \varepsilon, \omega_{b}\left(\kappa_{n_{\ell}-1}, \kappa_{m_{\ell}}\right)<\varepsilon$ and

$$
\begin{aligned}
& \frac{\varepsilon}{s^{2}} \leqslant \limsup _{n \rightarrow \infty} \omega_{b}\left(\tau_{m_{\ell}-1}, \tau_{n_{\ell}-1}\right) \leqslant \varepsilon s, \\
& \frac{\varepsilon}{s} \leqslant \limsup _{n \rightarrow \infty} \omega_{b}\left(\tau_{n_{\ell}-1}, \tau_{m_{\ell}}\right) \leqslant \varepsilon, \\
& \frac{\varepsilon}{s} \leqslant \limsup _{n \rightarrow \infty} \omega_{b}\left(\tau_{n_{\ell}}, \tau_{m_{\ell}-1}\right) \leqslant \varepsilon s^{2} .
\end{aligned}
$$


By the contractive condition (2), we obtain

$$
\begin{aligned}
\psi\left(2 s^{2} \omega_{b}\left(\tau_{m_{\ell}}, \tau_{n_{\ell}}\right)\right) & =\psi\left(2 s^{q} \omega_{b}\left(\tau_{m_{\ell}}, \tau_{n_{\ell}}\right)\right)=\psi\left(2 s^{q} \omega_{b}\left(T \kappa_{m_{\ell}}, T \kappa_{n_{\ell}}\right)\right) \\
& \leqslant \beta\left[\psi\left(M_{\omega_{b}}\left(\kappa_{m_{\ell}}, \kappa_{n_{\ell}}\right)\right)-\phi\left(M_{\omega_{b}}\left(\kappa_{m_{\ell}}, \kappa_{n_{\ell}}\right)\right)\right]
\end{aligned}
$$

where

$$
\begin{aligned}
& M_{\omega_{b}}\left(\kappa_{m_{\ell}}, \kappa_{n_{\ell}}\right)=\max \left\{\begin{array}{c}
\omega_{b}\left(S \kappa_{m_{\ell}}, S \kappa_{n_{\ell}}\right), \omega_{b}\left(S \kappa_{m_{\ell}}, T \kappa_{n_{\ell}}\right), \omega_{b}\left(S \kappa_{n_{\ell}}, T \kappa_{m_{\ell}}\right), \\
\frac{\omega_{b}\left(S \kappa_{m_{\ell}}, \kappa_{m_{\ell}}\right)+\omega_{b}\left(T \kappa_{n_{\ell}}, \kappa_{n_{\ell}}\right)}{4 s}
\end{array}\right\} \\
&=\max \left\{\begin{array}{c}
\omega_{b}\left(\tau_{m_{\ell}-1}, \tau_{n_{\ell}-1}\right), \omega_{b}\left(\tau_{m_{\ell}}-1, \tau_{n_{\ell}}\right), \omega_{b}\left(\tau_{n_{\ell}-1}, \tau_{m_{\ell}}\right), \\
\frac{\omega_{b}\left(\tau_{m_{\ell}-1}, \tau_{m_{\ell}}\right)+\omega_{b}\left(\tau_{n_{\ell}-1}, \tau_{n_{\ell}}\right)}{4 s}
\end{array}\right\} .
\end{aligned}
$$

Passing the upper limit as $k \rightarrow \infty$ in (9) and using (6) and (7), we can get

$$
\lim \sup _{n \rightarrow \infty} M_{\omega_{b}}\left(\kappa_{m_{\ell}}, \kappa_{n_{\ell}}\right)=\max \left\{\varepsilon s, \varepsilon s^{2}, \varepsilon, 0\right\} .
$$

Again, passing the upper limit as $k \rightarrow \infty$ in (8) and applying (10), we have

$$
\psi\left(2 s^{2} \varepsilon\right) \leqslant \beta\left[\psi\left(\varepsilon s^{2}\right)-\phi\left(\varepsilon s^{2}\right)\right] .
$$

By properties of $\psi, \phi$ and the assumption $\varepsilon>0$ leads to a contradiction. Hence the sequence $\left\{\tau_{n}\right\}$ is a Cauchy sequence in the complete $b$-metric-like space $\left(\Omega, \omega_{b}\right)$. By completeness, there is a point $\eta \in \Omega$ such that

$$
\lim _{n \rightarrow \infty} \omega_{b}\left(\tau_{n}, \eta\right)=\lim _{n \rightarrow \infty} \omega_{b}\left(T \kappa_{n}, \eta\right)=\lim _{n \rightarrow \infty} \omega_{b}\left(S \kappa_{n+1}, \eta\right)=0 .
$$

Since $T(\Omega) \subset S(\Omega)$, there exists $\xi \in \Omega$ such that $S \xi=\eta$. Now, we shall prove that $T \xi=S \xi$, then by (11)

$$
\begin{aligned}
\psi\left(2 s^{2} \omega_{b}(\eta, T \xi)\right)= & \psi\left(2 s^{q} \omega_{b}(\eta, T \xi)\right)=\psi\left(2 s^{q} \omega_{b}\left(T \kappa_{n}, T \xi\right)\right) \\
\leqslant & \beta \psi\left(\max \left\{\begin{array}{c}
\omega_{b}\left(S \kappa_{n}, S \xi\right), \omega_{b}\left(S \kappa_{n}, T \xi\right), \omega_{b}\left(S \xi, T \kappa_{n}\right), \\
\frac{\omega_{b}\left(S \kappa_{n}, T \kappa_{n}\right)+\omega_{b}(T \xi, S \xi)}{4 s}
\end{array}\right\}\right) \\
& -\beta \phi\left(\max \left\{\begin{array}{c}
\omega_{b}\left(S \kappa_{n}, S \xi\right), \omega_{b}\left(S \kappa_{n}, T \xi\right), \omega_{b}\left(S \xi, T \kappa_{n}\right), \\
\frac{\omega_{b}\left(S \kappa_{n}, T \kappa_{n}\right)+\omega_{b}(T \xi, S \xi)}{4 s}
\end{array}\right\}\right) \\
= & \beta \psi\left(\max \left\{\begin{array}{c}
\omega_{b}\left(\tau_{n-1}, S \xi\right), \omega_{b}\left(\tau_{n-1}, T \xi\right), \omega_{b}\left(S \xi, \tau_{n}\right), \\
\frac{\omega_{b}\left(\tau_{n-1}, \tau_{n}\right)+\omega_{b}(T \xi, S \xi)}{4 s}
\end{array}\right\}\right) \\
& -\beta \phi\left(\max \left\{\begin{array}{c}
\omega_{b}\left(\tau_{n-1}, S \xi\right), \omega_{b}\left(\tau_{n-1}, T \xi\right), \omega_{b}\left(S \xi, \tau_{n}\right), \\
\frac{\omega_{b}\left(\tau_{n-1}, \tau_{n}\right)+\omega_{b}(T \xi, S \xi)}{4 s}
\end{array}\right\}\right) .
\end{aligned}
$$

By taking the limit as $n \rightarrow \infty$ in (12) and using (6) and (11), we can write

$$
\begin{aligned}
\psi\left(2 s \omega_{b}(\eta, T \xi)\right) \leqslant & \psi\left(2 s^{2} \omega_{b}(\eta, T \xi)\right) \\
\leqslant & \beta \psi\left(\max \left\{\omega_{b}(\eta, S \xi), \omega_{b}(\eta, T \xi), \omega_{b}(S \xi, \eta), \frac{\omega_{b}(T \xi, \eta)+\omega_{b}(S \xi, \eta)}{4}\right\}\right) \\
& -\beta \phi\left(\max \left\{\omega_{b}(\eta, S \xi), \omega_{b}(\eta, T \xi), \omega_{b}(S \xi, \eta), \frac{\omega_{b}(T \xi, \eta)+\omega_{b}(S \xi, \eta)}{4}\right\}\right) \\
= & \beta\left\{\psi\left(2 s \omega_{b}(\eta, T \xi)\right)-\phi\left(\omega_{b}(\eta, T \xi)\right)\right\} \\
< & \psi\left(2 s \omega_{b}(\eta, T \xi)\right)-\phi\left(\omega_{b}(\eta, T \xi)\right) .
\end{aligned}
$$

By the definition of $\psi$ and $\phi$, we have a contradiction. Hence $\omega_{b}(\eta, T \xi)=0$, i.e.,

$$
\eta=T \xi=S \xi
$$


Then, $T$ and $S$ have a coincidence point $\eta \in \Omega$, then by Proposition $1, \eta$ is a unique common fixed point of the pair $(S, T)$, whenever, $T$ and $S$ are weakly compatible. This completes the proof.

As a consequence of Theorem 1, we obtain the following results.

Corollary 1. Let $\left(\Omega, \omega_{b}\right)$ be a complete $b$-metric-like space with parameter $s \geqslant 1$. If the nonlinear mappings $T, S: \Omega \rightarrow \Omega$ are weakly compatible such that $T(\Omega) \subset S(\Omega)$. Assume that $\psi \in \Psi, \phi \in \Phi, \beta \in\left[0, \frac{1}{2}\right)$ and $q \geqslant 2$ such that the condition

$$
\psi\left(2 s^{q} \omega_{b}(T \kappa, T \tau)\right) \leqslant \beta\left(\frac{\psi\left(M_{\omega_{b}}(\kappa, \tau)\right)}{1+\phi\left(M_{\omega_{b}}(\kappa, \tau)\right)}\right),
$$

holds for all $\kappa, \tau \in \Omega$, where $M(\kappa, \tau)$ is referred to in Definition 5 , therefore $T$ and $S$ have a unique common fixed point in $\Omega$.

Proof. The inequality (13) implies the inequality (2). So the proof finished by Theorem 1.

Corollary 2. Suppose that $\left(\Omega, \omega_{b}\right)$ is a complete $b$-metric-like space with coefficient $s \geqslant 1$. If the nonlinear mappings $T, S: \Omega \rightarrow \Omega$ are weakly compatible such that $T(\Omega) \subset S(\Omega)$. Assume that $\psi \in \Psi, \phi \in \Phi, \beta \in\left[0, \frac{1}{2}\right)$ and $q \geqslant 2$ such that the condition

$$
\psi\left(2 s^{q} \omega_{b}(T \kappa, T \tau)\right) \leqslant \beta\left(\frac{\psi\left(M_{\omega_{b}}(\kappa, \tau)\right) \phi\left(M_{\omega_{b}}(\kappa, \tau)\right)}{1+\phi\left(M_{\omega_{b}}(\kappa, \tau)\right)}\right)
$$

holds for all $\kappa, \tau \in \Omega$, where $M(\kappa, \tau)$ is mentioned in Definition 5 , therefore $T$ and $S$ have a unique common fixed point in $\Omega$.

Proof. The inequality (14) implies the inequality (2). Hence the conclusion follows from Theorem 1.

Corollary 3. Consider $\left(\Omega, \omega_{b}\right)$ is a complete b-metric-like space with coefficient $s \geqslant 1$. If the nonlinear mappings $T, S: \Omega \rightarrow \Omega$ are weakly compatible such that $T(\Omega) \subset S(\Omega)$. Assume that $\psi \in \Psi, \phi \in \Phi, \beta \in\left[0, \frac{1}{2}\right)$ and $q \geqslant 2$ such that the condition

$$
\psi\left(2 s^{q} \omega_{b}(T \kappa, T \tau)\right) \leqslant \beta\left(\frac{\psi\left(M_{\omega_{b}}(\kappa, \tau)\right)-\phi\left(M_{\omega_{b}}(\kappa, \tau)\right)}{1+\phi\left(M_{\omega_{b}}(\kappa, \tau)\right)}\right),
$$

holds for all $\kappa, \tau \in \Omega$, where $M(\kappa, \tau)$ is defined as in Definition 5 , therefore $T$ and $S$ have a unique common fixed point in $\Omega$.

Proof. Taking into account that $\phi$ is a lower semi-continuous function with $\phi(t)=0 \Leftrightarrow t=0$, we have

$$
\beta\left(\frac{\psi\left(M_{\omega_{b}}(\kappa, \tau)\right)-\phi\left(M_{\omega_{b}}(\kappa, \tau)\right)}{1+\phi\left(M_{\omega_{b}}(\kappa, \tau)\right)}\right) \leqslant \beta\left(\frac{\psi\left(M_{\omega_{b}}(\kappa, \tau)\right)}{1+\phi\left(M_{\omega_{b}}(\kappa, \tau)\right)}\right),
$$

for all $\kappa, \tau \in \Omega$ and $\beta \in\left[0, \frac{1}{2}\right)$. Hence inequality (15) implies inequality (13). Hence the conclusion follows from Theorem 1 .

In particular, by taking $\psi(t)=t$ and $S=T$ in Theorem 1 , we have the following result.

Corollary 4. Let $\left(\Omega, \omega_{b}\right)$ be a complete $b$-metric-like space with parameter $s \geqslant 1$, and $T: \Omega \rightarrow \Omega$ be a given self-mapping that satisfies

$$
s^{q} \omega_{b}(T \kappa, T \tau) \leqslant \beta\left(M_{\omega_{b}}(\kappa, \tau)-\phi\left(M_{\omega_{b}}(\kappa, \tau)\right)\right),
$$


where

$$
M_{\omega_{b}}(\kappa, \tau)=\max \left\{\omega_{b}(\kappa, \tau), \omega_{b}(\kappa, T \tau), \omega_{b}(\tau, T \kappa), \frac{\omega_{b}(\kappa, T \kappa)+\omega_{b}(\tau, T \tau)}{4 s}\right\},
$$

for some constants $\beta \in[0,1), q \geqslant 2$ and for all $\kappa, \tau \in \Omega$. Then $T$ has a unique fixed point.

Putting $\psi(t)=t$ and $\phi(t)=\frac{1}{2} t$ in the above theorem, we can get the following result.

Corollary 5. Let $\left(\Omega, \omega_{b}\right)$ be a complete b-metric-like space with parameter $s \geqslant 1$, and $T, S: \Omega \rightarrow \Omega$ be given self-mappings satisfies

$$
s^{q} \omega_{b}(T \kappa, T \tau) \leqslant \beta \max \left\{\omega_{b}(S \kappa, S \tau), \omega_{b}(S \kappa, T \tau), \omega_{b}(S \tau, T \tau), \frac{\omega_{b}(S \kappa, T \kappa)+\omega_{b}(T \tau, S \tau)}{4 s}\right\},
$$

for some constants $\beta \in\left[0, \frac{1}{2}\right), q \geqslant 2$ and for all $\kappa, \tau \in \Omega$. Then $T, S$ have a unique common fixed point, provided that the pair $(S, T)$ is weakly compatible.

By the relation $a+b \leqslant \max \{a, b\}$, we obtain the following result.

Corollary 6. Let $\left(\Omega, \omega_{b}\right)$ be a complete b-metric-like space with parameter $s \geqslant 1$. If $T, S: \Omega \rightarrow \Omega$ be self-mappings and there exists $q \geqslant 2$ and constants $c_{i} \geqslant 0, i=1, . ., 5$ with $c_{1}+c_{2}+c_{3}+c_{4}+c_{5}<1$ such that

$$
s^{q} \omega_{b}(T \kappa, T \tau) \leqslant c_{1} \omega_{b}(S \kappa, S \tau)+c_{2} \omega_{b}(S \kappa, T \tau)+c_{3} \omega_{b}(S \tau, T \tau)+c_{4} \omega_{b}(S \kappa, T \kappa)+c_{5} \omega_{b}(T \tau, S \tau),
$$

for all $\kappa, \tau \in \Omega$. Then $T$ and $S$ have a unique common fixed point, provided that the pair $(S, T)$ is weakly compatible.

The following examples illustrates the above results.

Example 8. Let $\Omega=[0,+\infty)$ and $\omega_{b}(\kappa, \tau)=\kappa^{2}+\tau^{2}+|\kappa-\tau|^{2}$ for all $\kappa, \tau \in \Omega$. It's obvious that $\omega_{b}$ is a b-metric like on $\Omega$, with coefficient $s=2$ and $\left(\Omega, \omega_{b}\right)$ is a complete. Define nonlinear self-mappings $T, S: \Omega \rightarrow \Omega$ by $T \kappa=\frac{1}{16} \ln \left(1+\frac{\kappa}{4}\right), S \kappa=\frac{1}{4} \ln \left(1+\frac{\kappa}{2}\right)$ for all $\kappa, \tau \in \Omega$, and the functions $\psi(t)=t, \phi(t)=\frac{t}{4}$ and constant $q=2$. It is clear that $T(\Omega) \subset S(\Omega)$. Since $t \geqslant \ln (1+t)$ for each $t \in[0, \infty)$, for all $\kappa, \tau \in \Omega$, we have

$$
\begin{aligned}
2 s^{2} \omega_{b}(T \kappa, T \tau) & =2 s^{2}\left(T^{2} \kappa+T^{2} \tau-|T \kappa-T \tau|^{2}\right) \\
& =8\left(\left(\frac{\ln \left(1+\frac{\kappa}{4}\right)}{16}\right)^{2}+\left(\frac{\ln \left(1+\frac{\tau}{4}\right)}{16}\right)^{2}+\left|\frac{\ln \left(1+\frac{\kappa}{4}\right)}{16}-\frac{\ln \left(1+\frac{\tau}{4}\right)}{16}\right|^{2}\right) \\
& \leqslant 8\left(\frac{\kappa^{2}}{16^{2}}+\frac{\tau^{2}}{16^{2}}+\left|\frac{\kappa}{16}-\frac{\tau}{16}\right|^{2}\right) \\
& =\frac{1}{32}\left(\kappa^{2}+\tau^{2}+|\kappa-\tau|^{2}\right)=\frac{1}{32} \omega_{b}(\kappa, \tau) .
\end{aligned}
$$

By the same method, we have $\omega_{b}(S \kappa, S \tau) \leqslant \frac{1}{16} \omega_{b}(\kappa, \tau)$, again

$$
\begin{aligned}
\omega_{b}(S \kappa, T \tau) & =\frac{\kappa^{2}}{16}+\frac{\tau^{2}}{16^{2}}+\left|\frac{\kappa}{4}-\frac{\tau}{16}\right|^{2} \\
& \leqslant \frac{\kappa^{2}}{16}+\frac{\tau^{2}}{16}+\left|\frac{\kappa}{4}-\frac{\tau}{4}\right|^{2}=\frac{1}{16}\left(\kappa^{2}+\tau^{2}+|\kappa-\tau|^{2}\right)=\frac{1}{16} \omega_{b}(\kappa, \tau) .
\end{aligned}
$$

Similarly, $\omega_{b}(S \tau, T \kappa)=\frac{1}{16} \omega_{b}(\kappa, \tau)$. On the other hand 


$$
\begin{aligned}
\omega_{b}(S \kappa, T \kappa) & =\left(S^{2} \kappa+S^{2} \kappa\right)=\frac{\kappa^{2}}{16}+\frac{\kappa^{2}}{16^{2}} \leqslant \frac{\kappa^{2}}{8}, \\
\omega_{b}(T \tau, S \tau) & =\left(T^{2} \tau+S^{2} \tau\right)=\frac{\tau^{2}}{16^{2}}+\frac{\tau^{2}}{16} \leqslant \frac{\tau^{2}}{8}, \\
\frac{\omega_{b}(S \kappa, T \kappa)+\omega_{b}(T \tau, S \tau)}{4 s} & \leqslant \frac{\frac{\kappa^{2}}{8}+\frac{\tau^{2}}{8}}{8}=\frac{\kappa^{2}+\tau^{2}}{64} \\
& \leqslant \frac{1}{64}\left(\kappa^{2}+\tau^{2}+|\kappa-\tau|^{2}\right)=\frac{1}{64} \omega_{b}(\kappa, \tau) .
\end{aligned}
$$

From definition $M_{\omega_{b}}(\kappa, \tau)$, we can write

$$
M_{\omega_{b}}(\kappa, \tau)=\frac{1}{16} \omega_{b}(\kappa, \tau)
$$

Combining (18) and (19), we get

$$
\begin{aligned}
\psi\left(2 s^{q} \omega_{b}(T \kappa, T \tau)\right) & =2 s^{2} \omega_{b}(T \kappa, T \tau)=\frac{1}{32} \omega_{b}(\kappa, \tau) \leqslant \frac{2}{3} \frac{3}{64} \omega_{b}(\kappa, \tau)=\frac{2}{3}\left(\frac{1}{16} \omega_{b}(\kappa, \tau)-\frac{1}{64} \omega_{b}(\kappa, \tau)\right) \\
& =\frac{2}{3}\left(M_{\omega_{b}}(\kappa, \tau)-\frac{1}{4} M_{\omega_{b}}(\kappa, \tau)\right)=\beta\left(\psi\left(M_{\omega_{b}}(\kappa, \tau)\right)-\phi\left(M_{\omega_{b}}(\kappa, \tau)\right)\right) .
\end{aligned}
$$

Therefore, the pair $(S, T)$ is $\beta_{q, \phi}^{s, \psi}$-contraction with $\beta=\frac{2}{3}<1$.

Moreover, the mappings $T$ and $S$ are weakly compatible, since $\frac{1}{16} \ln \left(1+\frac{\kappa}{4}\right)=\frac{1}{4} \ln \left(1+\frac{\kappa}{2}\right)$ only at $\kappa=0$ and $T 0=S 0=0$, also, TSO $=T 0=0=S T 0$. All of the axioms of Theorem 1 are satisfied and clearly $\kappa=0$ is a unique common fixed point of $T$ and $S$.

Example 9. Let $\Omega=\{0,1,2\}$. Define $\omega_{b}: \Omega \times \Omega \rightarrow[0,+\infty)$ as follows: $\omega_{b}(0,0)=0, \omega_{b}(1,1)=3$, $\omega_{b}(2,2)=1, \omega_{b}(0,1)=\omega_{b}(1,0)=8, \omega_{b}(0,2)=\omega_{b}(2,0)=1$ and $\omega_{b}(1,2)=\omega_{b}(2,1)=4$. Define $\phi(t)=\frac{t}{1+t}$, and define the mapping $T: \Omega \rightarrow \Omega$ by $T 0=0, T 1=2$, and $T 2=0$. It is obvious that $\left(\Omega, \omega_{b}\right)$ is a complete b-metric-like space with the constant $s=\frac{8}{5}$. Let $q=2$, we show that the condition (16) is true. Since

$$
\begin{aligned}
& s^{q} \omega_{b}(T 0, T 0)=0=\beta\left(M_{\omega_{b}}(0,0)-\phi\left(M_{\omega_{b}}(0,0)\right)\right), \beta=0 ; \\
& s^{q} \omega_{b}(T 0, T 1)=\frac{64}{25} \leqslant \frac{9}{25}\left(8-\frac{8}{9}\right)=\beta\left(M_{\omega_{b}}(0,1)-\phi\left(M_{\omega_{b}}(0,1)\right)\right), \beta=\frac{9}{25}<1 ; \\
& s^{q} \omega_{b}(T 0, T 2)=0=\beta\left(\frac{32}{5}-\frac{32}{37}\right)=\beta\left(M_{\omega_{b}}(0,2)-\phi\left(M_{\omega_{b}}(0,2)\right)\right), \beta=0 ; \\
& s^{q} \omega_{b}(T 1, T 1)=\frac{64}{25} \leqslant \frac{4}{5}\left(4-\frac{4}{5}\right)=\beta\left(M_{\omega_{b}}(1,1)-\phi\left(M_{\omega_{b}}(1,1)\right)\right), \beta=\frac{4}{5}<1 ; \\
& s^{q} \omega_{b}(T 1, T 2)=\frac{64}{25} \leqslant \frac{9}{25}\left(8-\frac{8}{9}\right)=\beta\left(M_{\omega_{b}}(1,2)-\phi\left(M_{\omega_{b}}(1,2)\right)\right), \beta=\frac{9}{25}<1 ; \\
& s^{q} \omega_{b}(T 2, T 2)=0=\beta\left(1-\frac{1}{2}\right)=\beta\left(M_{\omega_{b}}(2,2)-\phi\left(M_{\omega_{b}}(2,2)\right)\right), \beta=0,
\end{aligned}
$$

So, for all $\kappa, \tau \in \Omega$, we have $s^{q} \omega_{b}(T \kappa, T \tau) \leqslant \beta\left(M_{\omega_{b}}(\kappa, \tau)-\phi\left(M_{\omega_{b}}(\kappa, \tau)\right)\right)$. Therefore all the required hypotheses of Corollary 4 are satisfied, and thus we deduce the existence and uniqueness of the fixed point of $T$. Here, 0 is the unique fixed point of $T$.

\section{4. $(s, q)$-Graphic Contraction and Related Fixed Points}

In this section we use the contractive condition (17) of Corollary 6 to discuss some common fixed point results in the framework of $b$-metric-like spaces endowed with a graph.

In line with Jachymski [30], let $\left(\Omega, \omega_{b}\right)$ be a metric-like space and $\Re$ denote the diagonal of the Cartesian product $\Omega \times \Omega$. Consider a directed graph $G$ such that the set $\Theta(G)$ of its vertices coincides 
with $\Omega$, and the set $\Xi(G)$ of its edges contains all loops, i.e., $\Xi(G) \supseteq \Re$. We assume that $G$ has no parallel edges, so we can identify $G$ with the pair $(\Theta(G), \Xi(G))$. Moreover, we may treat $G$ as a weighted graph (see, [30]) by assigning to each edge the distance between its vertices.

By $G^{-1}$ we denote the conversion of a graph $G$, that is, the graph obtained from $G$ by reversing the direction of edges. Thus, we have

$$
\Xi\left(G^{-1}\right)=\{(\kappa, \tau) \in \Omega \times \Omega:(\tau, \kappa) \in \Xi(G)\} .
$$

The letter $\breve{G}$ denotes the undirected graph obtained from $G$ by ignoring the direction of edges. Actually, it will be more convenient for us to treat $\breve{G}$ as a directed graph for which the set of its edges is symmetric. Under this convention

$$
\Xi(\breve{G})=\Xi(G) \cup \Xi\left(G^{-1}\right) .
$$

If $\kappa$ and $\tau$ are vertices in a graph $G$, then a path in $G$ from $\kappa$ to $\tau$ of length $N(N \in \mathbb{N})$ is a sequence $\left\{\kappa_{i}\right\}_{i}^{N}=0$ of $N+1$ vertices such that $\kappa_{\circ}=\kappa, \kappa_{N}=\tau$ and $\left(\kappa_{n-1}, \kappa_{n}\right) \in \Xi(G)$ for $i=1, \ldots, N$. A graph $G$ is connected if there is a path between any two vertices. $G$ is weakly connected if $\breve{G}$ is connected.

Recently, some results have appeared providing sufficient conditions for a self mapping of $\Omega$ to be a Picard operator when $(\Omega, d)$ is endowed with a graph. The first result in this direction was given by Jachymski [30].

Definition 6. A nonlinear mapping $S: \Omega \rightarrow \Omega$ is a Banach $G$-contraction or simply $G$-contraction if $S$ preserves edges of $G$, i.e.,

$$
\forall \kappa, \tau \in \Omega:(\kappa, \tau) \in \Xi(G) \Rightarrow(S(\kappa), S(\tau)) \in \Xi(G)
$$

and $S$ decreases weights of edges of $G$ as for all $\kappa, \tau \in \Omega$, there exists $c \in(0,1)$, such that

$$
(\kappa, \tau) \in \Xi(G) \Rightarrow d(S(\kappa), S(\tau)) \leqslant c d(\kappa, \tau) .
$$

Throughout this section, we consider self-mappings $T, S: \Omega \rightarrow \Omega$ with $T(\Omega) \subset S(\Omega)$. Let $\mathcal{K}_{\circ} \in \Omega$ be an arbitrary point, then there exists $\kappa_{1} \in \Omega$ such that $T \kappa_{\circ}=S \kappa_{1}$. By repeating this step we can build a sequence $\left(S \kappa_{n}\right)$ such that $S \kappa_{n}=T \kappa_{n-1}$ and the following property:

The property $G_{\left(T, S \kappa_{n}\right)}$. If $\left(S \kappa_{n}\right)_{n \in N}$ is a sequence in $\Omega$ such that $S \kappa_{n} \rightarrow \kappa$ and $\left(S \kappa_{n}, S \kappa_{n+1}\right) \in E(\breve{G})$ for all $n \geqslant 1$, then there is a subsequence $\left(S \kappa_{n_{i}}\right)_{i \in N}$ of $\left(S \kappa_{n}\right)_{n \in N}$ such that $\left(S \kappa_{n_{i}}, \kappa\right) \in \Xi(\breve{G})$ for all $i \geqslant 1$. Here, we use the notion $G_{S T}=\left\{\kappa_{\circ} \in \Omega:\left(S \kappa_{n}, S \kappa_{m}\right) \in \Xi(\breve{G})\right.$, where $\left.m, n=1,2, \ldots\right\}$.

Now, we present the results of this section.

Theorem 2. Let $T, S: \Omega \rightarrow \Omega$ be self-mappings defined on a b-metric-like space $\left(\Omega, \omega_{b}\right)$ (with parameter $s \geqslant 1)$ endowed with a graph $G$, and satisfy (15) for all $\kappa, \tau \in \Omega$ with $(S \kappa, S \tau) \in \Xi(\breve{G})$ when either $\left(c_{1}+2 c_{2}+\right.$ $2 c_{3}+c_{4}+c_{5}<\frac{1}{s}$ and $\left.c_{3}+c_{4}<\frac{1}{s}\right)$ or $\left(c_{1}+c_{2}+c_{3}+2 c_{4}+2 c_{5}<\frac{1}{s}\right.$ and $\left.c_{1}+c_{3}<\frac{1}{s}\right)$.

Suppose that $T(\Omega) \subset S(\Omega)$ and $S(\Omega)$ is complete subspace of $\Omega$. Then

(i) If the property $G_{\left(T, S \kappa_{n}\right)}$ is satisfied and $G_{S T} \neq \varnothing$, then $T$ and $S$ have a point of coincidence in $\Omega$.

(ii) If $\kappa$ and $\tau$ are points of coincidence of $T$ and $S$, it implies $(\kappa, \tau) \in \Xi(\breve{G})$, then the point is a unique in $\Omega$, moreover, if the pair $(S, T)$ is weakly compatible, then $T$ and $S$ have a unique common fixed point in $\Omega$.

Proof. Assume that $G_{S T} \neq \varnothing$, there exists $\kappa_{\circ} \in G_{S T}$. Since $T(\Omega) \subset S(\Omega)$, there exists $\kappa_{1} \in \Omega$ such that $T \kappa_{\circ}=S \kappa_{1}$, again we can find $\kappa_{2} \in \Omega$ such that $T \kappa_{1}=S \kappa_{2}$. Repeat this step, we can build a sequence $S \kappa_{n}$ such that $S \kappa_{n}=T \kappa_{n-1}$ for $n=1,2, .$. , and $\left(S \kappa_{n}, S \kappa_{m}\right) \in \Xi(\breve{G})$. 
Suppose that $S \kappa_{n}=S \kappa_{n+1}$ for some $n \in N$. Then $S \kappa_{n}=T \kappa_{n}$, which leads to $\kappa_{n}$ is a coincidence point. So, we consider $S \kappa_{n} \neq S \kappa_{n+1}$ for all $n \in N$. By the condition (17), we can get

$$
\begin{aligned}
& s \omega_{b}\left(S \kappa_{n}, S \kappa_{n+1}\right) \\
\leqslant & s^{q} \omega_{b}\left(S \kappa_{n}, S \kappa_{n+1}\right)=s^{q} \omega_{b}\left(T \kappa_{n-1}, T \kappa_{n}\right) \\
\leqslant & c_{1} \omega_{b}\left(S \kappa_{n-1}, S \kappa_{n}\right)+c_{2} \omega_{b}\left(S \kappa_{n-1}, T \kappa_{n}\right)+c_{3} \omega_{b}\left(S \kappa_{n}, T \kappa_{n-1}\right) \\
& +c_{4} \omega_{b}\left(S \kappa_{n-1}, T \kappa_{n-1}\right)+c_{5} \omega_{b}\left(T \kappa_{n}, S \kappa_{n}\right) \\
\leqslant & c_{1} \omega_{b}\left(S \kappa_{n-1}, S \kappa_{n}\right)+c_{2} \omega_{b}\left(S \kappa_{n-1}, S \kappa_{n+1}\right)+c_{3} \omega_{b}\left(S \kappa_{n}, S \kappa_{n}\right) \\
& +c_{4} \omega_{b}\left(S \kappa_{n-1}, S \kappa_{n}\right)+c_{5} \omega_{b}\left(S \kappa_{n+1}, S \kappa_{n}\right) \\
\leqslant & c_{1} \omega_{b}\left(S \kappa_{n-1}, S \kappa_{n}\right)+s c_{2}\left[\omega_{b}\left(S \kappa_{n-1}, S \kappa_{n}\right)+\omega_{b}\left(S \kappa_{n}, S \kappa_{n+1}\right)\right]+2 s c_{3} \omega_{b}\left(S \kappa_{n-1}, S \kappa_{n}\right) \\
& +c_{4} \omega_{b}\left(S \kappa_{n-1}, S \kappa_{n}\right)+c_{5} \omega_{b}\left(S \kappa_{n+1}, S \kappa_{n}\right) .
\end{aligned}
$$

Thus, we have

$$
\omega_{b}\left(S \kappa_{n}, S \kappa_{n+1}\right) \leqslant\left(\frac{c_{1}+S c_{2}+2 s c_{3}+c_{4}}{1-s c_{2}-c_{5}}\right) \omega_{b}\left(S \kappa_{n-1}, S \kappa_{n}\right)=\lambda \omega_{b}\left(S \kappa_{n-1}, S \kappa_{n}\right) .
$$

where $\lambda=\frac{c_{1}+s c_{2}+2 s c_{3}+c_{4}}{1-s c_{2}-c_{5}}$. Since $c_{1}+2 s c_{2}+2 s c_{3}+c_{4}+c_{5}<s c_{1}+2 s c_{2}+2 s c_{3}+s c_{4}+s c_{5}<1$, we get $\lambda<1$. Continuing this process, we can write,

$$
\begin{aligned}
\omega_{b}\left(S \kappa_{n}, S \kappa_{n+1}\right) \leqslant & \lambda \omega_{b}\left(S \kappa_{n-1}, S \kappa_{n}\right) \\
\leqslant & \lambda^{2} \omega_{b}\left(S \kappa_{n-2}, S \kappa_{n-1}\right) \\
& \cdots \\
\leqslant & \lambda^{n} \omega_{b}\left(S \kappa_{\circ}, S \kappa_{1}\right) .
\end{aligned}
$$

Now, if $m>n$ for $m, n \in N$ and by $\left(\omega_{3}\right)$ of $b$-metric like conditions, one can write

$$
\begin{aligned}
\omega_{b}\left(S \kappa_{n}, S \kappa_{m}\right) \leqslant & s \omega_{b}\left(S \kappa_{n}, S \kappa_{n+1}\right)+s \omega_{b}\left(S \kappa_{n+1}, S \kappa_{m}\right) \\
\leqslant & s \omega_{b}\left(S \kappa_{n}, S \kappa_{n+1}\right)+s^{2} \omega_{b}\left(S \kappa_{n+1}, S \kappa_{n+2}\right)+s^{2} \omega_{b}\left(S \kappa_{n+2}, S \kappa_{m}\right) \\
& \ldots \\
\leqslant & s \omega_{b}\left(S \kappa_{n}, S \kappa_{n+1}\right)+s^{2} \omega_{b}\left(S \kappa_{n+1}, S \kappa_{n+2}\right)+s^{3} \omega_{b}\left(S \kappa_{n+2}, S \kappa_{n+3}\right)+\ldots \\
& +s^{m-n-1} \omega_{b}\left(S \kappa_{m-2}, S \kappa_{m-1}\right)+s^{m-n} \omega_{b}\left(S \kappa_{m-1}, S \kappa_{m}\right) .
\end{aligned}
$$

Hence, Equations (20) and (21) gives

$$
\begin{aligned}
\omega_{b}\left(S \kappa_{n}, S \kappa_{m}\right) \leqslant & s \lambda^{n} \omega_{b}\left(S \kappa_{\circ}, S \kappa_{1}\right)+s^{2} \lambda^{n+1} \omega_{b}\left(S \kappa_{\circ}, S \kappa_{1}\right)+s^{3} \lambda^{n+2} \omega_{b}\left(S \kappa_{\circ}, S \kappa_{1}\right)+\ldots \\
& +\lambda^{m-1} s^{m-n} \omega_{b}\left(S \kappa_{\circ}, S \kappa_{1}\right) \\
= & s \lambda^{n}\left(1+(s \lambda)+(s \lambda)^{2}+\ldots+(s \lambda)^{m-n-1}\right) \omega_{b}\left(S \kappa_{\circ}, S \kappa_{1}\right) \\
= & s \lambda^{n} \sum_{j=0}^{\infty}(s \lambda)^{j} \omega_{b}\left(S \kappa_{\circ}, S \kappa_{1}\right) \\
= & s \lambda^{n}\left(\frac{1}{1-s \lambda}\right) \omega_{b}\left(S \kappa_{\circ}, S \kappa_{1}\right) \rightarrow 0, \text { as } n \rightarrow \infty .
\end{aligned}
$$

Therefore, $\left(S \kappa_{n}\right)$ is a Cauchy sequence. The completeness of $S(\Omega)$ leads to, there is $\eta \in S(\Omega)$ such that $\left(S \kappa_{n}\right) \rightarrow \eta=S(\xi)$ for some $\xi \in \Omega$. As, $\kappa_{\circ} \in G_{S T}$, this implies that $\left(S \kappa_{n}, S \kappa_{m}\right) \in \Xi(\breve{G})$ for $n, m=1,2, \ldots$ and so, $\left(S \kappa_{n}, S \kappa_{n+1}\right) \in E(\breve{G})$. By property $G_{\left(T, S \kappa_{n}\right)}$, there is a subsequence $\left(S \kappa_{n_{i}}\right)_{i \in N}$ of $\left(S \kappa_{n}\right)_{n \in N}$ such that $\left(S \kappa_{n_{i}}, \eta\right) \in \Xi(\breve{G})$. Applying $\left(\omega_{3}\right)$ of $b$-metric like axioms, we can get

$$
\omega_{b}(T \xi, S \xi) \leqslant \omega_{b}\left(T \xi, T \kappa_{n_{i}}\right)+\omega_{b}\left(T \kappa_{n_{i}}, S \xi\right) .
$$


On the other hand, condition (17) gives

$$
\begin{aligned}
\omega_{b}\left(T \xi, T \kappa_{n_{i}}\right) \leqslant & s^{q} \omega_{b}\left(T \xi, T \kappa_{n_{i}}\right) \\
\leqslant & c_{1} \omega_{b}\left(S \xi, S \kappa_{n_{i}}\right)+c_{2} \omega_{b}\left(S \xi, T \kappa_{n_{i}}\right)+c_{3} \omega_{b}\left(S \kappa_{n_{i}}, T \xi\right) \\
& +c_{4} \omega_{b}(S \xi, T \xi)+c_{5} \omega_{b}\left(T \kappa_{n_{i}}, S \kappa_{n_{i}}\right) .
\end{aligned}
$$

Applying (23) in (22), we obtain that

$$
\begin{aligned}
\omega_{b}(T \xi, S \xi) \leqslant & c_{1} \omega_{b}\left(S \xi, S \kappa_{n_{i}}\right)+c_{2} \omega_{b}\left(S \xi, T \kappa_{n_{i}}\right)+c_{3} \omega_{b}\left(S \kappa_{n_{i}}, T \xi\right)+c_{4} \omega_{b}(S \xi, T \xi) \\
& +c_{5} \omega_{b}\left(T \kappa_{n_{i}}, S \kappa_{n_{i}}\right)+s \omega_{b}\left(T \kappa_{n_{i}}, S \xi\right) .
\end{aligned}
$$

Replacing $T \kappa_{n_{i}}$ with $S \kappa_{n_{i}+1}$ in (24), we get

$$
\begin{aligned}
\omega_{b}(T \xi, S \xi) \leqslant & c_{1} \omega_{b}\left(S \xi, S \kappa_{n_{i}}\right)+c_{2} \omega_{b}\left(S \xi, S \kappa_{n_{i}+1}\right)+c_{3} \omega_{b}\left(S \kappa_{n_{i}}, T \xi\right)+c_{4} \omega_{b}(S \xi, T \xi) \\
& +c_{5} \omega_{b}\left(S \kappa_{n_{i}+1}, S \kappa_{n_{i}}\right)+s \omega_{b}\left(S \kappa_{n_{i}+1}, S \xi\right) .
\end{aligned}
$$

Hence

$$
\begin{aligned}
\omega_{b}(T \xi, S \xi) \leqslant & \left(\frac{c_{1}}{1-c_{4}}\right) \omega_{b}\left(S \xi, S \kappa_{n_{i}}\right)+\left(\frac{c_{2}+s}{1-c_{4}}\right)\left(S \xi, S \kappa_{n_{i}+1}\right)+\left(\frac{c_{3}}{1-c_{4}}\right) \omega_{b}\left(S \kappa_{n_{i}}, T \xi\right) \\
& +\left(\frac{c_{5}}{1-c_{4}}\right) \omega_{b}\left(S \kappa_{n_{i}+1}, S \kappa_{n_{i}}\right) \\
\leqslant & \left(\frac{c_{1}}{1-c_{4}}\right) \omega_{b}\left(S \xi, S \kappa_{n_{i}}\right)+\left(\frac{c_{2}+s}{1-c_{4}}\right)\left(S \xi, S \kappa_{n_{i}+1}\right)+\left(\frac{s c_{3}}{1-c_{4}}\right) \omega_{b}\left(S \kappa_{n_{i}}, S \xi\right) \\
& +\left(\frac{s c_{3}}{1-c_{4}}\right) \omega_{b}(S \xi, T \xi)+\left(\frac{c_{5}}{1-c_{4}}\right) \lambda^{n_{i}} \omega_{b}\left(S \kappa_{1}, S \kappa_{\circ}\right) .
\end{aligned}
$$

Applying the limit of (25) as $i \rightarrow \infty$ and by $\lim _{i \rightarrow \infty} \omega_{b}\left(S \kappa_{n_{i}}, S \xi\right)=0$, we can get

$$
\omega_{b}(T \xi, S \xi) \leqslant\left(\frac{S c_{3}}{1-c_{4}}\right) \omega_{b}(S \xi, T \xi) .
$$

Since $\frac{s c_{3}}{1-c_{4}}<1$, it implies that $\omega_{b}(T \xi, S \xi)=0$. Therefore, $T \xi=S \xi=\eta$, and so $\xi$ is a coincidence point of $T$ and $S$, and $\eta$ is a point of coincidence.

For uniqueness, suppose that the axiom (ii) of Theorem 2 is satisfied, i.e., there is $\eta^{*} \in \Omega$ such that $T \zeta=S \zeta=\eta^{*}$ for some $\zeta \in \Omega$ and $\left(\eta, \eta^{*}\right) \in \Xi(\breve{G})$. Now, the condition (17) gives

$$
\begin{aligned}
s \omega_{b}\left(\eta, \eta^{*}\right) \leqslant & s^{q} \omega_{b}\left(\eta, \eta^{*}\right)=s^{q} \omega_{b}(T \xi, T \zeta) \leqslant c_{1} \omega_{b}(S \xi, S \zeta)+c_{2} \omega_{b}(S \xi, T \zeta)+c_{3} \omega_{b}(S \zeta, T \xi) \\
& +c_{4} \omega_{b}(S \xi, T \xi)+c_{5} \omega_{b}(T \zeta, S \zeta) \\
= & c_{1} \omega_{b}\left(\eta, \eta^{*}\right)+c_{2} \omega_{b}\left(\eta, \eta^{*}\right)+c_{3} \omega_{b}\left(\eta^{*}, \eta\right)+c_{4} \omega_{b}(\eta, \eta)+c_{5} \omega_{b}(\eta, \eta) \\
\leqslant & \left(c_{1}+c_{2}+c_{3}+2 s c_{4}+2 s c_{5}\right) \omega_{b}\left(\eta^{*}, \eta\right) .
\end{aligned}
$$

Hence, Equation (26) becomes $\omega_{b}\left(\eta, \eta^{*}\right) \leqslant \frac{1}{s^{2}} \omega_{b}\left(\eta, \eta^{*}\right)$, which gives that $\eta=\eta^{*}$, since $c_{1}+c_{2}+$ $c_{3}+2 c_{4}+2 c_{5}<s c_{1}+s c_{2}+s c_{3}+2 s c_{4}+2 s c_{5}<1$.

If $T$ and $S$ are weakly compatible, then by Proposition $1, T$ and $S$ have a unique common fixed point.

Now, By specifying some of the constants $c_{1}, c_{2}, c_{3}, c_{4}$ and $c_{5}$ of Theorem 2, we conclude the following results.

Corollary 7. Let $T, S: \Omega \rightarrow \Omega$ be self-mappings defined on a b-metric-like space $\left(\Omega, \omega_{b}\right)$ (with parameter $s \geqslant 1$ ) endowed with a graph $G$, and satisfy one of the following 


$$
\begin{aligned}
& s^{q} \omega_{b}(T \kappa, T \tau) \leqslant c_{1} \omega_{b}(S \kappa, S \tau), c_{1}<\frac{1}{s^{\prime}} \\
& s^{q} \omega_{b}(T \kappa, T \tau) \leqslant c_{4} \omega_{b}(S \kappa, T \kappa)+c_{5} \omega_{b}(T \tau, S \tau), c_{4}+c_{5}<\frac{1}{s}, \\
& s^{q} \omega_{b}(T \kappa, T \tau) \leqslant c_{2} \omega_{b}(S \kappa, T \tau)+c_{3} \omega_{b}(S \tau, T \kappa)+c_{4} \omega_{b}(S \kappa, T \kappa), c_{2}+c_{3}+c_{4}<\frac{1}{s},
\end{aligned}
$$

for all $\kappa, \tau \in \Omega$ with $(S \kappa, S \tau) \in \Xi(\breve{G})$. If the conditions of Theorem 2 hold, then $T$ and $S$ have a unique common fixed point in $\Omega$.

We can generalize Theorem 2 to integral and exponential type as follows.

Corollary 8. Let $T, S: \Omega \rightarrow \Omega$ be self-mappings defined on a b-metric-like space $\left(\Omega, \omega_{b}\right)$ (with parameter $s \geqslant 1$ ) endowed with a graph $G$, and satisfy

$$
\begin{aligned}
s^{q} \int_{0}^{\omega_{b}(T \kappa, T \tau)} \lambda(\rho) d \rho \leqslant c_{1} & \int_{0}^{\omega_{b}(S \kappa, S \tau)} \lambda(\rho) d \rho+c_{2} \int_{0}^{\omega_{b}(S \kappa, T \tau)} \lambda(\rho) d \rho \\
& +c_{3} \int_{0}^{\omega_{b}(S \tau, T \kappa)} \lambda(\rho) d \rho+c_{4} \int_{0}^{\omega_{b}(S \kappa, T \kappa)} \lambda(\rho) d \rho+c_{5} \int_{0}^{\omega_{b}(T \tau, S \tau),} \lambda(\rho) d \rho,
\end{aligned}
$$

for all $\kappa, \tau \in \Omega$ with $(S \kappa, S \tau) \in \Xi(\breve{G})$ when either $\left(c_{1}+2 c_{2}+2 c_{3}+c_{4}+c_{5}<\frac{1}{s}\right.$ and $\left.c_{3}+c_{4}<\frac{1}{s}\right)$ or $\left(c_{1}+c_{2}+c_{3}+2 c_{4}+2 c_{5}<\frac{1}{s}\right.$ and $\left.c_{1}+c_{3}<\frac{1}{s}\right)$, where $\lambda:[0, \infty) \rightarrow[0, \infty)$ is a Lebesgue-integrable mapping satisfying $\int_{0}^{\epsilon} \lambda(\rho) d \rho>0$ for $\epsilon>0$.

Suppose that $T(\Omega) \subset S(\Omega)$ and $S(\Omega)$ is complete subspace of $\Omega$. If the conditions of Theorem 2 hold, then $T$ and $S$ have a unique common fixed point in $\Omega$.

Corollary 9. Let $T, S: \Omega \rightarrow \Omega$ be self-mappings defined on a b-metric-like space $\left(\Omega, \omega_{b}\right)$ (with parameter $s \geqslant 1$ ) endowed with a graph $G$, and satisfy

$$
s^{q} e^{\omega_{b}(T \kappa, T \tau)} \leqslant c_{1} e^{\omega_{b}(S \kappa, S \tau)}+c_{2} e^{\omega_{b}(S \kappa, T \tau)}+c_{3} e^{\omega_{b}(S \tau, T \kappa)}+c_{4} e^{\omega_{b}(S \kappa, T \tau)}+c_{5} e^{\omega_{b}(T \tau, S \tau)},
$$

for all $\kappa, \tau \in \Omega$ with $(S \kappa, S \tau) \in \Xi(\breve{G})$ when either $\left(c_{1}+2 c_{2}+2 c_{3}+c_{4}+c_{5}<\frac{1}{s}\right.$ and $\left.c_{3}+c_{4}<\frac{1}{s}\right)$ or $\left(c_{1}+c_{2}+c_{3}+2 c_{4}+2 c_{5}<\frac{1}{s}\right.$ and $\left.c_{1}+c_{3}<\frac{1}{s}\right)$, where $e:[0, \infty) \rightarrow[0, \infty)$ is a Lebesgue-integrable mapping satisfying $e^{\epsilon}>0$ for $\epsilon>0$. Then $T$ and $S$ have a unique common fixed point in $\Omega$, whenever the conditions of Theorem 2 are satisfied.

Note that, the mappings $T$ and $S$ satisfying condition (17) only on a graph $G$. To explain that, we give the following example.

Example 10. Let $\Omega=[0,+\infty), q=2, s=2$ and $T, S: \Omega \rightarrow \Omega$ be nonlinear mappings such that

$$
T \kappa=\left\{\begin{array}{cl}
\frac{1}{3} \kappa^{3}, & \text { if } \quad \kappa \neq \sqrt{8} \\
0, & \text { if } \quad \kappa=\sqrt{8}
\end{array} \text { and } S \kappa=\left\{\begin{array}{cl}
\kappa^{3}, & \text { if } \quad \kappa \neq 2 \\
1, & \text { if } \quad \kappa=2
\end{array}\right. \text {. }\right.
$$

Let $\left(\Omega, \omega_{b}\right)$ be a b-metric-like space under the distance $\omega_{b}(\kappa, \kappa)=(\kappa+\tau)^{2}, G$ be the graph with $\Theta(G)=\Omega$ and $\Xi(G)=\{(\kappa, \kappa): \kappa \in \Omega\} \cup\left\{\left(0, \frac{1}{3^{n}}\right): n \in \mathbb{N}\right\}$ and the constants $c_{1}=\frac{1}{3}, c_{2}=c_{4}=\frac{1}{128}, c_{3}=\frac{1}{9}$ and $c_{5}=\frac{1}{64}$ such that $c_{1}+c_{2}+c_{3}+2 c_{4}+2 c_{5}<\frac{1}{s}$ and $c_{1}+c_{3}<\frac{1}{s}$.

Note that $(S \kappa, S \tau) \in \Xi(\breve{G})$ only occurs in two cases: 
Case 1. $\kappa=\tau$;

Case 2. For some $n \in \mathbb{N}$, either $\kappa=\tau=0$ and the other one is $\frac{1}{\sqrt{3^{\left(\frac{n}{3}\right)}}}$.

Now, if $\kappa=\tau=0$, then $\omega_{b}(T \kappa, T \tau)=\omega_{b}(0,0)=0$ which satisfies condition (17). Next, for some $n \in \mathbb{N}$, assume that $\kappa=0$ and $\tau=\frac{1}{\sqrt{3^{\left(\frac{n}{3}\right)}}}$, without loss of generality, we can get

$$
\begin{aligned}
& \omega_{b}(S \kappa, S \tau)=\omega_{b}\left(0, \frac{1}{\sqrt{3^{n}}}\right)=\frac{9}{3^{n+2}}, \quad \omega_{b}(S \kappa, T \tau)=\omega_{b}\left(0, \frac{1}{\sqrt{3^{n+2}}}\right)=\frac{1}{3^{n+2}} \\
& \omega_{b}(S \tau, T \kappa)=\omega_{b}\left(\frac{1}{\sqrt{3^{n}}}, 0\right)=\frac{9}{3^{n+2}}, \quad \omega_{b}(S \kappa, T \kappa)=\omega_{b}(0,0)=0 \\
& \omega_{b}(T \tau, S \tau)=\omega_{b}\left(\frac{1}{\sqrt{3^{n+2}}}, \frac{1}{\sqrt{3^{n}}}\right)=\frac{16}{3^{n+2}}, \quad \omega_{b}(T \kappa, T \tau)=\omega_{b}\left(0, \frac{1}{\sqrt{3^{n+2}}}\right)=\frac{1}{3^{n+2}} .
\end{aligned}
$$

Now,

$$
\begin{aligned}
& c_{1} \omega_{b}(S \kappa, S \tau)+c_{2} \omega_{b}(S \kappa, T \tau)+c_{3} \omega_{b}(S \tau, T \kappa)+c_{4} \omega_{b}(S \kappa, T \kappa)+c_{5} \omega_{b}(T \tau, S \tau) \\
= & \frac{1}{3}\left(\frac{9}{3^{n+2}}\right)+\frac{1}{64}\left(\frac{1}{3^{n+2}}\right)+\frac{1}{9}\left(\frac{9}{3^{n+2}}\right)+\left(\frac{1}{128}\right)(0)+\frac{1}{64}\left(\frac{16}{3^{n+2}}\right) \\
= & \frac{1}{3^{n+2}}\left(3+\frac{1}{64}+1+\frac{1}{4}\right)>2^{2} \frac{1}{2^{n+1}}=s^{q} \omega_{b}(T \kappa, T \tau) .
\end{aligned}
$$

Otherwise, let $\kappa=0$ and $\tau=2$. Then $(S \kappa, S \tau)=(0,1) \notin \Xi(\breve{G})$ and

$$
\begin{aligned}
& \omega_{b}(S \kappa, S \tau)=\omega_{b}(0,1)=1, \quad \omega_{b}(S \kappa, T \tau)=\omega_{b}\left(0, \frac{8}{3}\right)=\frac{64}{9} \\
& \omega_{b}(S \tau, T \kappa)=\omega_{b}(1,0)=1, \quad \omega_{b}(S \kappa, T \kappa)=\omega_{b}(0,0)=0 \\
& \omega_{b}(T \tau, S \tau)=\omega_{b}\left(\frac{8}{3}, 1\right)=\frac{121}{9}, \quad \omega_{b}(T \kappa, T \tau)=\omega_{b}\left(0, \frac{8}{3}\right)=\frac{64}{9} .
\end{aligned}
$$

Thus,

$$
\begin{aligned}
& c_{1} \omega_{b}(S \kappa, S \tau)+c_{2} \omega_{b}(S \kappa, T \tau)+c_{3} \omega_{b}(S \tau, T \kappa)+c_{4} \omega_{b}(S \kappa, T \kappa)+c_{5} \omega_{b}(T \tau, S \tau) \\
= & \frac{1}{3}(1)+\frac{1}{64}\left(\frac{64}{9}\right)+\frac{1}{9}(1)+\frac{1}{128}(0)+\frac{1}{64}\left(\frac{121}{9}\right) \\
= & \frac{49}{64}<2^{2} \frac{64}{9}=\omega_{b}(T \kappa, T \tau) .
\end{aligned}
$$

Hence, $T$ and S satisfy our condition (17) on the graph $G$ but do not on the whole space $\Omega$.

To justify Theorem 2, we discuss the following example.

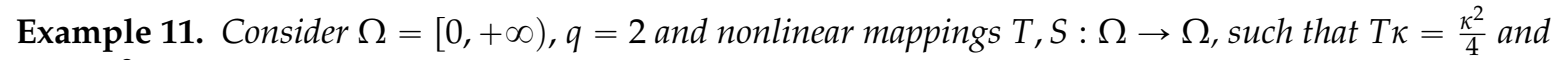
$S \kappa=\kappa^{2}$. Assume that $\left(\Omega, \omega_{b}\right)$ is a b-metric-like space under the same distance of Example 10, $G$ is a graph with $\Theta(G)=\Omega, \Xi(G)=\{(\kappa, \kappa): \kappa \in \Omega\} \cup\left\{\left(0, \frac{1}{2^{n}}\right): n \in \mathbb{N}\right\}$, and the constants of $(15)$ are $c_{1}=c_{3}=\frac{1}{8}$ and $c_{2}=c_{4}=c_{5}=\frac{1}{64}$ such that $c_{1}+c_{2}+c_{3}+2 c_{4}+2 c_{5}<\frac{1}{s}$ and $c_{1}+c_{3}<\frac{1}{s}$. It is obvious that $T(\Omega) \subset S(\Omega)$ and $S(\Omega)$ is complete subspace of $\Omega$. The pair $(S \kappa, S \tau) \in \Xi(\breve{G})$ only occurs in two cases:

Case 1. $\kappa=\tau$;

- for some $n \in \mathbb{N}$;

Case 2. Either $\kappa=\tau=0$ and the other one is $\frac{1}{2^{\left(\frac{n}{2}\right)}}$.

Now, if $\kappa=\tau=0$, then $\omega_{b}(T \kappa, T \tau)=\omega_{b}(0,0)=0$ which satisfies condition (17). Next, for some $n \in \mathbb{N}$, assume that $\kappa=0$ and $\tau=\frac{1}{2^{\left(\frac{n}{2}\right)}}$, then 


$$
\begin{aligned}
& \omega_{b}(S \kappa, S \tau)=\omega_{b}\left(0, \frac{1}{2^{n+2}}\right)=\frac{16}{4^{n+2}}, \quad \omega_{b}(S \kappa, T \tau)=\omega_{b}\left(0, \frac{1}{2^{n+2}}\right)=\frac{1}{4^{n+2}} \\
& \omega_{b}(S \tau, T \kappa)=\omega_{b}\left(\frac{1}{2^{n}}, 0\right)=\frac{16}{4^{n+2}}, \quad \omega_{b}(S \kappa, T \kappa)=\omega_{b}(0,0)=0 \\
& \omega_{b}(T \tau, S \tau)=\omega_{b}\left(\frac{1}{2^{n+2}}, \frac{1}{2^{n}}\right)=\frac{25}{4^{n+2}}, \quad \omega_{b}(T \kappa, T \tau)=\omega_{b}\left(0, \frac{1}{2^{n+2}}\right)=\frac{1}{4^{n+2}} .
\end{aligned}
$$

Now,

$$
\begin{aligned}
& c_{1} \omega_{b}(S \kappa, S \tau)+c_{2} \omega_{b}(S \kappa, T \tau)+c_{3} \omega_{b}(S \tau, T \kappa)+c_{4} \omega_{b}(S \kappa, T \kappa)+c_{5} \omega_{b}(T \tau, S \tau) \\
= & \frac{1}{8}\left(\frac{16}{4^{n+2}}\right)+\frac{1}{64}\left(\frac{1}{4^{n+2}}\right)+\frac{1}{8}\left(\frac{16}{4^{n+2}}\right)+\left(\frac{1}{64}\right)(0)+\frac{1}{64}\left(\frac{25}{4^{n+2}}\right) \\
= & \frac{1}{4^{n+2}}\left(2+\frac{1}{64}+2+\frac{25}{64}\right) \geqslant 2^{2} \frac{1}{4^{n+2}}=s^{q} \omega_{b}(T \kappa, T \tau) .
\end{aligned}
$$

So, for all $\kappa, \tau \in \Omega$ with the pair $(S \kappa, S \tau) \in \Xi(\breve{G})$, the condition (17) of Corollary 6 is verified.

At the last, let $\kappa_{\circ} \in \Omega$. If $\kappa_{\circ}=0$, it is easy to show that the pair $\left(S \kappa_{n}, S \kappa_{m}\right)=(0,0) \in \Xi(\breve{G})$ for $m$ $n=1,2, \ldots G_{S T} \neq \varnothing$.

For $\kappa_{\circ} \neq 0$, there is $\kappa_{1} \in \Omega$ such that $S \kappa_{1}=T \kappa_{\circ}=\frac{x_{\circ}^{2}}{4}$ which implies $\kappa_{1}=\frac{\kappa_{\circ}}{2}$. Similarly, there is $\kappa_{2} \in \Omega$ such that $S \kappa_{2}=T \kappa_{1}=\frac{\kappa_{0}^{2}}{4^{2}}$, hence $\kappa_{2}=\frac{\kappa_{\circ}}{2^{2}}$. Repeat these steps, we can built the sequence $\left(S \kappa_{n}\right)$ such that $S \kappa_{n}=T \kappa_{n-1}=\frac{\kappa_{\circ}^{2}}{4^{n}}$.

It is clear that $\left(S \kappa_{n}, S \kappa_{m}\right)=\left(\frac{\kappa_{\circ}^{2}}{4^{n}}, \frac{\kappa_{\circ}^{2}}{4^{m}}\right) \notin \Xi(\breve{G})$. Thus, the constant sequence $S \kappa_{n}=0$ is only convergent sequence such that $\left(S \kappa_{n}, S \kappa_{m}\right) \in E(\breve{G})$. So for every subsequence $\left(S \kappa_{n_{i}}\right)$ of $\left(S \kappa_{n}\right)$, we have $\left(S \kappa_{n_{i}}, 0\right) \in E(\breve{G})$.

Also, the mappings $T$ and $S$ are weakly compatible at $\kappa=0$ and TSO $=T 0=0=S T 0$, so all conditions of Theorem 2 are satisfied and 0 is the unique common fixed point of $T$ and $S$ in $\Omega$.

In order to clarify the importance of the property $G_{\left(T, S x_{n}\right)}$, we present an example as follows:

Example 12. Let $\Omega=[0,1], s=q=2$ and $S, T: \Omega \rightarrow \Omega$, such that

$$
T x=\left\{\begin{array}{ll}
\frac{\kappa^{4}}{64}, & \text { if } \quad x \neq 0 \\
\frac{1}{16}, & \text { if } \quad x=0
\end{array} \text { and } S x=\left\{\begin{array}{ll}
\frac{\kappa^{2}}{2}, & \text { if } x \neq 0 \\
1, & \text { if } x=0
\end{array}\right. \text {. }\right.
$$

Assume that $\left(\Omega, \omega_{b}\right)$ is a b-metric-like space under the same distance of Example 10, $G$ be the graph with $\Theta(G)=\Omega$ and $\Xi(G)=\{(0,0)\} \cup\{(\kappa, \tau) \in(0,1] \times(0,1]\}$, and the constants $c_{1}=c_{2}=c_{3}=c_{4}=c_{5}=\frac{1}{64}$. It is obvious that $T(\Omega) \subset S(\Omega)$ and $S(\Omega)$ is complete subspace of $\Omega$. The pair $(S \kappa, S \tau) \in \Xi(\breve{G})$ only occurs in two cases:

Case 1. $\kappa=\tau=0$;

Case 2. $\kappa \neq 0 \neq \tau$.

If $\kappa=\tau=0$, then

$$
\begin{aligned}
S^{q} \omega_{b}(T \kappa, T \tau) & =\frac{4}{64} \leqslant \frac{1}{64} \omega_{b}(S \kappa, S \tau)=c_{1} \omega_{b}(S \kappa, S \tau) \\
& \leqslant \frac{1}{64} \omega_{b}(S \kappa, S \tau)+\frac{1}{64} \omega_{b}(S \kappa, T \tau)+\frac{1}{64} \omega_{b}(S \tau, T \kappa)+\frac{1}{64} \omega_{b}(S \kappa, T \kappa)+\frac{1}{64} \omega_{b}(T \tau, S \tau) .
\end{aligned}
$$

If $x \neq 0$ and $y \neq 0$, then

$$
\begin{aligned}
s^{q} \omega_{b}(T \kappa, T \tau) & =4\left(\frac{\kappa^{4}}{64}+\frac{\tau^{4}}{64}\right)^{2}=\frac{1}{1024}\left(\kappa^{4}+\tau^{4}\right)\left(\kappa^{4}+\tau^{4}\right) \\
& \leqslant \frac{1}{512}\left(\kappa^{4}+\tau^{4}\right) \leqslant \frac{1}{256}\left(\kappa^{4}+\tau^{4}\right) \leqslant \frac{1}{64} \frac{1}{4}\left(\kappa^{4}+\tau^{4}+2 \kappa^{2} \tau^{2}\right)=c_{1} \omega_{b}(S \kappa, S \tau) \\
& \leqslant \frac{1}{64} \omega_{b}(S \kappa, S \tau)+\frac{1}{64} \omega_{b}(S \kappa, T \tau)+\frac{1}{64} \omega_{b}(S \tau, T \kappa)+\frac{1}{64} \omega_{b}(S \kappa, T \kappa)+\frac{1}{64} \omega_{b}(T \tau, S \tau)
\end{aligned}
$$


Let $\kappa_{\circ} \in(0,1]$, then $S \kappa_{1}=T \kappa_{\circ}=\frac{\kappa_{0}^{4}}{64} \neq 0$, which leads to $\kappa_{1}=\frac{\kappa_{\circ}^{2}}{4 \sqrt{2}} \in(0,1]$. By the same step, we can find $\kappa_{2} \in(0,1]$ such that $S \kappa_{2}=T \kappa_{1}$. Repeating the same steps, we can get $S \kappa_{n}=T \kappa_{n-1} \neq 0$. Therefore $\left(S \kappa_{n}, S \kappa_{m}\right) \in \Xi(\breve{G})$. As the above results $\kappa_{\circ} \in G_{S T}$, and so $G_{S T} \neq \varnothing$.

Now, let $\kappa_{\circ}=1$. We will obtain a sequence $\left(S \kappa_{n}\right)$ by $S \kappa_{n}=T \kappa_{n-1}$. So, $S \kappa_{1}=T \kappa_{\circ}=\frac{1}{64}$, hence $\kappa_{1}=\frac{\sqrt{2}}{8}$. Similarly, there is $\kappa_{2}$ such that $S \kappa_{2}=T \kappa_{1}=\frac{(\sqrt{2})^{4}}{8^{6}}$, thus $\kappa_{2}=\frac{(\sqrt{2})^{3}}{8^{3}}$. Repeating this process, we get

$$
S \kappa_{n}=T \kappa_{n-1}=\frac{(\sqrt{2})^{2^{n+1}-4}}{8^{2^{n+1}-2}} \rightarrow 0 \text { as } n \rightarrow \infty .
$$

So, $\left(S \kappa_{n}, S \kappa_{m}\right) \in E(\breve{G})$ and $S \kappa_{n} \rightarrow 0$ but $\left(S \kappa_{n}, 0\right) \notin \Xi(\breve{G})$. So there is no subsequence $\left(S \kappa_{n_{i}}\right)$ of $\left(S \kappa_{n}\right)$ such that $\left(S \kappa_{n_{i}}, 0\right) \in \Xi(\breve{G})$. Also, we can easily see that the mappings $T$ and $S$ have no coincidence point, so there is no common fixed point.

\section{An Application to Nonlinear Integral Equation}

In this section, we will use Corollary 1 to find an analytical solution of the following nonlinear integral equation:

$$
\eta(\rho)=\int_{0}^{A} \Lambda(\rho, \theta) \sigma(\theta, \eta(\theta)) d \theta ;(\rho, \theta) \in[0, A]^{2} .
$$

Let $\Omega=C([0, A], \mathbb{R})$ be the set of real continuous functions defined on $[0, A]$ for $A>0$, endowed with

$$
\omega_{b}(\kappa, \tau)=\max _{\rho \in[0,1]}(|\kappa(\rho)|+|\tau(\rho)|)^{m} \text { for all } \kappa, \tau \in \Omega,
$$

where $m>1$. It is clear that $\left(\Omega, \omega_{b}\right)$ is a complete $b$-metric-like space with parameter $s=2^{m-1}$.

Consider a nonlinear self-mapping $T: \Omega \rightarrow \Omega$ given by

$$
\operatorname{T\eta }(\rho)=\int_{0}^{A} \Lambda(\rho, \theta) \sigma(\theta, \eta(\theta)) d \theta .
$$

Theorem 3. Suppose that Equation (27) with the following axioms:

(i) $\Lambda:[0, A] \times[0, A] \rightarrow[0, \infty)$ is a continuous function;

(ii) $\sigma:[0, A] \times \mathbb{R} \rightarrow \mathbb{R}$, where $\sigma(\theta,$.$) is monotone nondecreasing mapping for all \theta \in[0, A]$;

(iii) $\sup _{\rho, \theta \in[0, A]} \int_{0}^{A} \Lambda(\rho, \theta) d \theta \leqslant 1$;

(iv) there exists a constant $\mu \in(0,1)$ such that for all $(\rho, \theta) \in[0, A]^{2}$ and $\kappa, \tau \in \mathbb{R}$,

$$
\mid \sigma(\theta, \kappa(\theta))+\sigma\left(\theta, \tau(\theta) \mid \leqslant\left(\frac{\mu}{3 s^{3}}\right)^{\frac{1}{m}} \Lambda(\rho, \theta)(|\kappa(\theta)|+|\tau(\theta)|) .\right.
$$

Then a nonlinear integral Equation (27) has a unique solution $\kappa \in \Omega$.

Proof. For $\kappa, \tau \in \Omega$, from conditions (iii) and (iv), for all $\theta$ and $\rho$, we get 


$$
\begin{aligned}
s^{2} \omega_{b}(T \kappa(\rho), T \tau(\rho)) & =s^{2}(|T \kappa(\rho)|+|T \tau(\rho)|)^{m} \\
& =s^{2}\left(\left|\int_{0}^{A} \Lambda(\rho, \theta) \sigma(\theta, \kappa(\theta)) d \theta\right|+\left|\int_{0}^{A} \Lambda(\rho, \theta) \sigma(\theta, \tau(\theta)) d \theta\right|\right)^{m} \\
& \leqslant s^{2}\left(\int_{0}^{A}|\Lambda(\rho, \theta) \sigma(\theta, \kappa(\theta))| d \theta+\int_{0}^{A}|\Lambda(\rho, \theta) \sigma(\theta, \tau(\theta))| d \theta\right)^{m} \\
& \left.\leqslant s^{2}\left(\int_{0}^{A}\left(\frac{\mu}{3 s^{3}}\right)^{\frac{1}{m}} \Lambda(\rho, \theta)\left(((|\kappa(\theta)|+\mid \tau(\theta)) \mid)^{m}\right)^{\frac{1}{m}}\right) d \theta\right)^{m} \\
& \left.\leqslant s^{2}\left(\int_{0}^{A}\left(\frac{\mu}{3 s^{3}}\right)^{\frac{1}{m}} \Lambda(\rho, \theta)\left(\omega_{b}^{\frac{1}{m}}(\kappa(\theta), \tau(\theta))\right)\right) d \theta\right)^{m} \\
& \left.\leqslant s^{2}\left(\frac{\mu}{3 s^{3}}\right) \omega_{b}(\kappa(\theta), \tau(\theta))\right)\left(\int_{0}^{A} \Lambda(\rho, \theta) d \theta\right)^{m} \\
& \left.\leqslant \frac{\mu}{3 s} \omega_{b}(\kappa(\theta), \tau(\theta))\right) \leqslant \frac{\mu}{3 s} M_{\omega_{b}}(\kappa, \tau)=\frac{1}{3}\left(M_{\omega_{b}}(\kappa, \tau)-\left(1-\frac{\mu}{s}\right) M_{\omega_{b}}(\kappa, \tau)\right) \\
& =\frac{1}{3}\left(M_{\omega_{b}}(\kappa, \tau)-\phi M_{\omega_{b}}(\kappa, \tau)\right) .
\end{aligned}
$$

Therefore, all the axioms of Corollary 1 are satisfied by taking the coefficient $q=2$, and function $\phi(\kappa)=\left(1-\frac{\mu}{s}\right) \kappa$, where $\frac{\mu}{s} \in(0,1)$ and $\beta=\frac{1}{3}$. Hence the mapping $T$ has a unique fixed point in $X$, which is a solution of the integral equation in (27).

An example to illustrate the requirements of Theorem 3 is presented as follows.

Example 13. Consider the following nonlinear integral equation

$$
\eta(\rho)=\frac{1}{48 s} \int_{0}^{1} \theta^{2} \eta(\theta) d \theta, \rho \in[0,1] .
$$

Then it has a solution in $\Omega=(C[0,1], \mathbb{R})$.

Proof. Let $T: \Omega \rightarrow \Omega$ be defined as $\operatorname{T} \eta(\rho)=\frac{1}{48 s} \int_{0}^{1} \theta^{2} \eta(\theta) d \theta$. By specifying $\Lambda(\rho, \theta)=\frac{\theta}{4}, f(\theta, \eta(\theta))=$ $\frac{\theta \eta(\theta)}{12 s}$ in Theorem 3, we can write

(i) the function $\Lambda(\rho, \theta)$ is continuous on $[0,1] \times[0,1]$,

(ii) $\sigma(\theta, \eta(\theta))$ is monotone increasing on $[0,1] \times \mathbb{R}$ for all $\theta \in[0,1]$,

(iii)

$$
\sup _{\rho, \theta \in[0, A]} \int_{0}^{A} \Lambda(\eta, \theta) d \theta=\sup _{\rho \in[0,1]} \int_{0}^{1} \frac{\theta}{4} d \theta \leqslant \sup _{\rho \in[0,1]} \frac{1}{8}=\frac{1}{8}<1,
$$

(iv) By taking $m=3$, so there exists a constant $\mu=\frac{1}{9} \in(0,1)$ such that for all $(\rho, \theta) \in[0,1]^{2}$ and $\kappa, \tau \in \mathbb{R}$, we have

$$
\begin{aligned}
\mid \sigma(\theta, \kappa(\theta))+\sigma(\theta, \tau(\theta) \mid & =\frac{\theta}{12 s}|\kappa(\theta)+\tau(\theta)| \leqslant \frac{1}{12}\left(\frac{\theta}{s}\right)(|\kappa(s)|+|\tau(s)|) \\
& =\left(\frac{\mu}{3 s^{3}}\right)^{\frac{1}{3}} \frac{\theta}{4}(|\kappa(s)|+|\tau(s)|)=\left(\frac{\mu}{3 s^{3}}\right)^{\frac{1}{m}} \Lambda(\rho, \theta)(|\kappa(\theta)|+|\tau(\theta)|) .
\end{aligned}
$$


Therefore, the conditions of Theorem 3 are justified, hence a nonlinear mapping $T$ has a fixed point in $\Omega$, which is a solution to Equation (28).

\section{Conclusions}

The analytical solution of nonlinear integral equations and graph theory are important applications in fixed point theory, where they have attracted the interest of many authors in academic research. Continuing in this direction, this article presents some common fixed point theorems for a pair of $\beta_{q, \phi}^{s, \psi}$-contractive mappings in $b$-metric-like spaces. Our results extend and generalize the results of [27] in two mappings and [31] in $b$-metric-like spaces and other spaces. It can be pointed out that the obtained results could be extended to some open mathematical problems in the fields of convergence of trajectory solutions to the equilibrium points and stability of dynamic systems and to those related ones formulated in the fractal space. It is also of interest to investigate the convergence properties in problems described by operators firstly defined on infinite, dimensional Hilbert spaces which are then represented in truncated finite dimensional ones. See, for instance, refs. [32-36] and some references therein. Next, some common fixed point results in the framework of $b$-metric-like spaces endowed with a graph have been discussed. Moreover, some important examples are given to support our results and we showed the existence of a solution for a nonlinear integral equation.

Author Contributions: All authors contributed equally and significantly in writing this article. All authors read and approved the final manuscript.

Funding: This research received funding from the Spanish Government and the European Commission through Grant RTI2018-094336-B-I00 (MINECO/FEDER, UE).

Acknowledgments: The authors are grateful to the Spanish Government and the European Commission for Grant RTI2018-094336-B-I00 (MINECO/FEDER, UE).

Conflicts of Interest: The authors declare that they have no competing interests concerning the publication of this article.

\section{References}

1. Berzig, M.; Chandok, S.; Khan, M.S. Generalized Krasnoselskii fixed point theorem involving auxiliary functions in bimetric spaces and application to two-point boundary value problem. Appl. Math. Comput. 2014, 248, 323-327. [CrossRef]

2. Gopal, D.; Abbas, M.; Vetro, C. Some new fixed point theorems in manger PM-spaces with application to Volterra type integral equation. Appl. Math. Comput. 2014, 232, 955-967.

3. Liu, Z.; Li, X.; Minkan, S.; Cho, S.Y. Fixed point theorems for mappings satisfying contractive condition of integral type and applications. Fixed Point Theory Appl. 2011, 64, 1-18. [CrossRef]

4. Pathak, H.K.; Khan, M.S.; Tiwari, R. A common fixed point theorem and its application to nonlinear integral equations. Comput. Math. Appl. 2007, 53, 961-971. [CrossRef]

5. Shahzad, N.; Valero, O.; Alghamdi, M.A. A fixed point theorem in partial quasi-metric spaces and an application to software engineering. Appl. Math. Comput. 2015, 268, 1292-1301. [CrossRef]

6. Hussain, N.; Taoudi, M.A. Krasnoselskii-type fixed point theorems with applications to Volterra integral equations. Fixed Point Theory Appl. 2013, 2013, 1-16.

7. Bakhtin, I.A. The contraction mapping principle in quasi-metric spaces. Funct. Anal. Ulianowsk Gos. Ped. Inst. 1989, 30, 26-37.

8. Czerwik, S. Contraction mappings in b-metric spaces. Acta Math. Inform. Univ. Ostrav. 1993, 1, 5-11.

9. Yamaod, O.; Sintunavarat, W.; Je Cho, Y. Existence of a common solution for a system of nonlinear integral equations via fixed point methods in $b$-metric spaces. Open Math. 2017, 14, 128-145. [CrossRef]

10. Aydi, H. $\alpha$-implicit contractive pair of mappings on quasi $b$-metric spaces and an application to integral equations. J. Nonlinear Convex Anal. 2016, 17, 2417-2433.

11. Mustafa, Z.; Roshan, J.R.; Parvaneh, V.; Kadelburg, Z. Some common fixed point results in ordered $b$-metric spaces. J. Inequal. Appl. 2013, 2013, 1-26. [CrossRef]

12. Sarwar, M.; Rahman, M. Fixed point theorems for Ciric's and generalized contractions in $b$-metric spaces. Int. J. Anal. Appl. 2015, 7, 70-78. 
13. Pacurar, M. Sequences of almost contractions and fixed points in $b$-metric spaces. Anal. Univ. Vest Timis. Ser. Mat-Inform. 2010, 48, 125-137.

14. Roshan, J.R.; Parvaneh, V.; Altun, I. Some coincidence point results in ordered $b$-metric spaces and applications in a system of integral equations. Appl. Math. Comput. 2014, 262, 725-737.

15. Roshan, J.R.; Parvaneh, V.; Sedghi, S.; Shobkolaei, N.; Shatanawi, W. Common fixed points of almost generalized $(\psi, \phi)$-contractive mappings in ordered b-metric spaces. Fixed Point Theory Appl. 2013, 2013, 1-23.

16. Harandi, A.A. Metric-like spaces, partial metric spaces and fixed points. Fixed Point Theory Appl. 2012, 2012, 1-10.

17. Hussain, N.; Roshan, J.R.; Parvaneh, V.; Kadelburg, Z. Fixed points of contractive mappings in $b$-metric-like spaces. Sci. World J. 2014, 2014, 1-15. [CrossRef]

18. Aydi, A.; Felhi, A.; Sahmim, S. Common fixed points via implicit contractions on $b$-metric-like spaces. J. Nonlinear Sci. Appl. 2017, 10, 1524-1537. [CrossRef]

19. Nashine, H.K.; Kadelburg, Z. Existence of solutions of Cantilever Beam Problem via $\alpha-\beta-F G-$ contractions in $b$-metric-like spaces. Filomat 2017, 31, 3057-3074. [CrossRef]

20. Aydi, H.; Felhi, A.; Sahmim, S. Ćirić-Berinde fixed point theorems for multi-valued mappings on $\alpha$ complete metric-like spaces. Filomat 2017, 31, 3727-3740. [CrossRef]

21. Aydi, H.; Felhi, A.; Afshari, H. New Geraghty type contractions on metric-like spaces. J. Nonlinear Sci. Appl. 2017, 10, 780-788. [CrossRef]

22. Aydi, H.; Karapinar, E. Fixed point results for generalized $\alpha-\psi$-contractions in metric-like spaces and applications. Electron. J. Differ. Equ. 2015, 133, 1-15.

23. Alsulami, H.; Gülyaz, S.; Karapinar, E.; Erha, I.M. An Ulam stability result on quasi- $b$-metric-like spaces. Open Math. 2016, 14, 1-15. [CrossRef]

24. Hammad, H.A.; De la Sen, M. Solution of nonlinear integral equation via fixed point of cyclic $\alpha_{L}^{\psi}-$ rational contraction mappings in metric-like spaces. Bull. Braz. Math. Soc. New Ser. 2019. [CrossRef]

25. Alghmandi, M.A.; Hussain, N.; Salimi, P. Fixed point and coupled fixed point theorems on $b$-metric-like spaces. J. Inequal. Appl. 2013, 402, 1-25.

26. Aydi, H.; Felhi, A.; Sahmim, S. On common fixed points for $(\alpha, \psi)$-contractions and generalized cyclic contractions in $b$-metric-like spaces and consequences. J. Nonlinear Sci. Appl. 2016, 9, 2492-2510. [CrossRef]

27. Zoto, Z.; Radenovic, S.; Ansari, A.H. On some fixed point results for $(s, p, \alpha)$-contractive mappings in b-metric-like spaces and applications to integral equations. Open Math. 2018, 16, 235-249. [CrossRef]

28. Jungck, G. Common fixed points for noncontinuous nonself maps on nonmetric spaces. Far East J. Math. Sci. 1996, 4, 199-215.

29. Abbas, M.; Jungck, G. Common fixed point results for noncommuting mappings without continuity in cone metric spaces. J. Math. Anal. Appl. 2008, 341, 416-420. [CrossRef]

30. Jachymski, J. The contraction principle for mappings on a metric space with a graph. Proc. Am. Math. Soc. 2008, 1, 1359-1373. [CrossRef]

31. Mustafa, Z.; Jaradata, M.M.M.; Jaradatb, H.M. Some common fixed point results of graphs on $b$-metric space. J. Nonlinear Sci. Appl. 2016, 9, 4838-4851. [CrossRef]

32. Nash, J.F., Jr.; Rassias, M.T. (Eds.) Open Problems in Mathematics; Springer: New York, NY, USA, 2016.

33. Hutchinson, J.E. Fractals and self similarity. Indiana Univ. Math. J. 1981, 30, 713-747. [CrossRef]

34. Guariglia, E. Primality, Fractality and image analysis. Entropy 2009, 21, 304. [CrossRef]

35. Ri, S.I. A new fixed point theorem in the fractal space. Indag. Math.-New Ser. 2016, 27, 85-93. [CrossRef]

36. De la Sen, M. On contractive and convergence properties of a class of truncated operators represented through Schauder bases with applications. Indag. Math.-New Ser. 2015, 26, 431-444. [CrossRef]

(C) 2019 by the authors. Licensee MDPI, Basel, Switzerland. This article is an open access article distributed under the terms and conditions of the Creative Commons Attribution (CC BY) license (http:/ / creativecommons.org/licenses/by/4.0/). 